\title{
THE COMPUTATIONAL COMPLEXITY OF PLETHYSM COEFFICIENTS
}

\section{Nick Fischer And Christian IKenmeyer}

\begin{abstract}
In two papers, Bürgisser and Ikenmeyer (STOC 2011, STOC 2013) used an adaption of the geometric complexity theory (GCT) approach by Mulmuley and Sohoni (Siam J Comput 2001, 2008) to prove lower bounds on the border rank of the matrix multiplication tensor. A key ingredient was information about certain Kronecker coefficients. While tensors are an interesting test bed for GCT ideas, the far-away goal is the separation of algebraic complexity classes. The role of the Kronecker coefficients in that setting is taken by the so-called plethysm coefficients: These are the multiplicities in the coordinate rings of spaces of polynomials. Even though several hardness results for Kronecker coefficients are known, there are almost no results about the complexity of computing the plethysm coefficients or even deciding their positivity.
\end{abstract}

In this paper, we show that deciding positivity of plethysm coefficients is NP-hard and that computing plethysm coefficients is \#P-hard. In fact, both problems remain hard even if the inner parameter of the plethysm coefficient is fixed. In this way, we obtain an inner versus outer contrast: If the outer parameter of the plethysm coefficient is fixed, then the plethysm coefficient can be computed in polynomial time.

Moreover, we derive new lower and upper bounds and in special cases even combinatorial descriptions for plethysm coefficients, which we consider to be of independent interest. Our technique uses discrete tomography in a more refined way than the recent work on Kronecker coefficients by Ikenmeyer, Mulmuley, and Walter (Comput Compl 2017). This makes our work the first to apply techniques from discrete tomog- 
raphy to the study of plethysm coefficients. Quite surprisingly, that interpretation also leads to new equalities between certain plethysm coefficients and Kronecker coefficients.

Keywords. Geometric complexity theory, Plethysm coefficients, Computational complexity, NP-hardness.

Subject classification. 68Q17, 05E10.

\section{Introduction}

Geometric complexity theory (GCT) is an approach toward the separation of Valiant's algebraic complexity classes using algebraic geometry and representation theory. These ideas were introduced by Mulmuley \& Sohoni (2001, 2008). A first implementation of that framework proves lower bounds on the border rank of the matrix multiplication tensor (Bürgisser \& Ikenmeyer 2011; Bürgisser \& Ikenmeyer 2013). In these papers, Kronecker coefficients play a key role, as they are the multiplicities in the coordinate rings of spaces of tensors. While that line of research turns out as an interesting test case for GCT ideas, the ultimate goal remains to separate algebraic complexity classes, that is, certain sets of (families of) polynomials. Switching from tensors to polynomials, the role of Kronecker coefficients in Bürgisser \& Ikenmeyer (2011); Bürgisser \& Ikenmeyer (2013) is now taken by plethysm coefficients (see e.g., Bläser \& Ikenmeyer 2018, Sec. 12.4(i)). Plethysm coefficients are the main subject of "GCT7" (Mulmuley 2007), and they also appear prominently in the GCT publications (Bürgisser 2016; Bürgisser et al. 2017; Kadish \& Landsberg 2014; Kumar 2015).

The main subject of study in this paper are plethysm coefficients and, to a lesser extent, Kronecker coefficients. Both types of representation theoretic coefficients are not only important in GCT, but they are fundamental objects in algebraic combinatorics and invariant theory. They describe the dimensions of invariant spaces whose study dates back to the XIX ${ }^{\text {th }}$ century mathematicians Aronhold, Cayley, Clebsch, Gordan, and Sylvester, to name a few. Hermite's reciprocity theorem (Hermite 1854) is a famous early special case of Foulkes' positivity conjecture on differences of 
plethysm coefficients, see (Foulkes 1950). In 1999, Richard Stanley highlighted the quest for a combinatorial description for plethysm coefficients as Problem 9 in his survey on "outstanding open problems" in algebraic combinatorics (Stanley 1999b); finding a combinatorial interpretation for Kronecker coefficients is Problem 10 in the same paper ${ }^{1}$. Even though GCT asks to compare these (and related) coefficients (see e.g., Ikenmeyer 2012, Appendix), both coefficients have been studied mostly independently. But this has started to change recently, as can be seen for example from the fact that the Fall 2016 Eastern Sectional Meeting of the American Mathematical Society held a special session on "Plethysm and Kronecker products in Representation Theory" and also from the 2020 Oberwolfach Mini-Workshop on "Kronecker, Plethysm, and Sylow Branching Coefficients and their applications to Complexity Theory." To the best of our knowledge, the only papers that give inequalities between these two sorts of coefficients are Bürgisser et al. (2019); Ikenmeyer \& Panova (2017); Manivel (2011)2.

In GCT, both coefficients do not only serve as the multiplicities in the coordinate rings of spaces of tensors or polynomials, but they also appear as terms in nonnegative formulas that describe multiplicities of coordinate rings of important group orbits: The plethysm coefficients appear as terms in the nonnegative formulas for the multiplicities in the coordinate ring of the orbit of the product of variables (Landsberg 2017, Sec. 9.2.3), the power sum polynomial (Ikenmeyer \& Kandasamy 2020), the unit tensor (Ikenmeyer 2019, Sec. 3.7), and the permanent polynomial (Bürgisser et al. 2011, Eq. 5.5.2), whereas the Kronecker coefficients appear as terms in the nonnegative formulas for the multiplicities in the coordinate ring of the orbit of the determinant polynomial (Bürgisser

\footnotetext{
${ }^{1}$ In the language of computational complexity theory, "finding a combinatorial description" is commonly interpreted as "proving that the function that computes the coefficient from its parameter list is in the complexity class \#P." Mulmuley (2007) lists conjectures that would imply the containment in \#P, thus proving Stanley's Problem 9.

${ }^{2}$ Mulmuley (2009) writes that "The Kronecker coefficients [...] are [...] special cases of the fundamental plethysm constants"; however, that definition of "plethysm constants" is much broader than the plethysm coefficients that we study in this paper.
} 
et al. 2011, Eq. 5.2.6), the permanent polynomial (Bürgisser et al. 2011, Eq. 5.5.2, both the plethysm coefficients and the Kronecker coefficients appear in this formula), the matrix multiplication tensor (Bürgisser \& Ikenmeyer 2011, Thm. 5.3), the unit tensor (Ikenmeyer 2019, Sec. 3.7, both the plethysm coefficients and the Kronecker coefficients appear in this formula), and the trace of the matrix power polynomial (Gesmundo et al. 2017, Thm 2.10). In theoretical physics, the plethysm and Kronecker coefficients are closely related to the quantum marginal problem for indistinguishable and distinguishable particles, respectively, see e.g., Christandl et al. (2007) and Christandl et al. (2014, Sec. 7), where the asymptotic positivity of the coefficients is studied.

While the \#P-hardness of the computation of the Kronecker coefficient dates back to Bürgisser \& Ikenmeyer (2008) and Briand et al. (2009), the recent paper by Ikenmeyer et al. (2017) proves the NP-hardness of deciding positivity of the Kronecker coefficient. This was a setback for the GCT program, as it was originally conjectured that Kronecker positivity would be decidable in polynomial time, much in the same way as the well-known Littlewood-Richardson coefficients: Even though the computation of Littlewood-Richardson coefficients is \#P-complete (Narayanan 2006), positivity of Littlewood-Richardson coefficients can be decided in polynomial time (De Loera \& McAllister 2006) using linear programming (see also Mulmuley et al. 2012) and even with a combinatorial max-flow algorithm (Bürgisser \& Ikenmeyer 2013). A similarly efficient algorithm for Kronecker positivity would have made the search for obstructions (i.e., inequalities between coefficients) much easier.

In this paper, we prove NP-hardness of deciding positivity of plethysm coefficients (Theorem 3.5) and \#P-hardness of the computation of plethysm coefficients (Theorem 3.6). Not even the \#Phardness of the computation of plethysm coefficients was known prior to our work. Indeed, our reduction is quite subtle compared to the one used to prove NP-hardness of deciding positivity of Kronecker coefficients Ikenmeyer et al. (2017).

The GCT no-go results (Bürgisser et al. 2019; Ikenmeyer \& Panova 2017) hold only in so-called padded settings, which includes 
the original Mulmuley-Sohoni approach. Not much is known about unpadded settings, but first results were obtained by Gesmundo et al. (2017). Our results show that even in the unpadded settings comparing multiplicities or finding GCT occurrence obstructions will be challenging. However, recent results show that it is possible to circumvent this hardness, see Ikenmeyer \& Kandasamy (2020).

Structure of the Paper. Section 2 explains the necessary background from representation theory. Section 3 states our main hardness results and puts them in contrast to what is known about efficiently computable subcases. Section 4 is a first important step in our reduction: We show that the inner parameter of the plethysm coefficient can be fixed to be three. Section 5 translates an interesting subcase of the plethysm coefficient problem into a problem in discrete tomography. In Section 6, we use this tomography description to design a sequence of reductions that finally prove our hardness result. Sections 7 and 8 contain results of independent interest: Section 7 highlights new close connections to Kronecker coefficients, while Section 8 contains more combinatorial descriptions for the plethysm coefficients. Finally, Section 9 uses classical results to provide an algorithm that places the problem of computing plethysm coefficients in the complexity class GapP.

\section{Preliminaries from Representation Theory}

The definition of plethysm and Kronecker coefficients that we use requires some elementary algebraic geometry and representation theory, as can be found for example in the standard textbooks (Fulton \& Harris 1991; Kraft 1985; Sagan 2001). The necessary material can also for example be found in Landsberg (2011, Ch. 6).

A composition is a list of nonnegative integers $\lambda=\left(\lambda_{0}, \lambda_{1}, \ldots\right)$, which we always treat as finite by omitting trailing zeros. If the entries are nonincreasing, then we call $\lambda$ a partition. A partition $\lambda$ is often presented as its Young diagram, which is a top-left justified array containing $\lambda_{i}$ boxes in the $i$-th row. For instance, $(3,1)$ is identified with the Young diagram $\mathrm{C}^{\square}$. In that presentation, it makes sense to term $\lambda_{0}$ the width of $\lambda$ and the number of nonzero entries the height of $\lambda$, denoted by $\ell(\lambda)$. We refer to the number 
$|\lambda|:=\sum_{i} \lambda_{i}$ of boxes in $\lambda^{\prime}$ 's Young diagram as the size of $\lambda$. Finally, let the transpose $\lambda^{t}$ of $\lambda$ denote the partition obtained by exchanging rows and columns. For example, $(3,1)^{t}=(2,1,1)$ is depicted as

Let $V, W$ be a finite-dimensional complex vector spaces. We say that $V$ is a $G$-representation for some group $G$ if there is a group homomorphism $\rho: G \rightarrow \mathrm{GL}(V)$. For notational convenience, we will never mention $\rho$ explicitly and instead write $g v:=\rho(g)(v)$. As an important example, there is a canonical action of the general linear group $\mathrm{GL}(V)$ on $V$ given by $g v:=g(v)$, for all $g \in \mathrm{GL}(V)$ and $v \in V$, and thus $V$ can be seen as a $\mathrm{GL}(V)$-representation.

Assume that $W$ is a $\mathrm{GL}(V)$-representation (for instance $W=V$, as in the previous paragraph). If $\operatorname{diag}\left(\alpha_{0}, \ldots, \alpha_{k}\right) w=\alpha_{0}^{\lambda_{0}} \cdots \alpha_{k}^{\lambda_{k}} w$ for some composition $\lambda$, then we say that $w$ is a weight vector of weight $\lambda$. Note that for all matrices $m \in \mathfrak{g l}(V):=\mathbb{C}^{\operatorname{dim} V \times \operatorname{dim} V}$ we have that $\mathrm{Id}+\varepsilon m \in \mathrm{GL}(V)$ for all small enough $\varepsilon$, where Id is the $\operatorname{dim} V \times \operatorname{dim} V$ identity matrix. We say that $W$ admits an action of the Lie-algebra $\mathfrak{g l}(V)$ defined by $m w:=\lim _{\varepsilon \rightarrow 0} \varepsilon^{-1}((\operatorname{Id}+\varepsilon m) w-w)$ for all $m \in \mathfrak{g l}(V), w \in W$. For $i<j$, define the raising operator $E_{i, j} \in \mathfrak{g l}(V)$ as the matrix with a single one at position $(i, j)$ and zeros everywhere else. A weight vector $w$ of weight $\lambda$ is called a highest weight vector if $w$ vanishes under the action of all raising operators. In that case, $\lambda$ is guaranteed to be a partition. It is well-known that the irreducible polynomial representations of $\mathrm{GL}(V)$ are characterized by their unique (up to scale) highest weight vectors, which in turn, are indexed by partitions of height at most $\operatorname{dim} V$. The irreducible $\mathrm{GL}(V)$-representation of type $\nu$ is called the Schur module and denoted by $S^{\nu} V$. The Schur module $S^{\nu} V$ can be explicitly realized as a subrepresentation of any $\mathrm{GL}(V)$-representation by taking the linear span of the $\mathrm{GL}(V)$-orbit of a highest weight vector of weight $\nu$, provided such a vector exists. More generally, for any $\mathrm{GL}(V)$-representation $W$, $S^{\nu} W$ is constructed as follows via character theory. There is a natural linear $\mathrm{GL}(V)$-action on its $m$-th tensor power $\otimes^{m} W$ given by $g\left(w_{1} \otimes w_{2} \otimes \cdots \otimes w_{m}\right):=\left(g w_{1}\right) \otimes\left(g w_{2}\right) \otimes \cdots \otimes\left(g w_{m}\right)$ and linear continuation. We define $S^{\nu} W$ to be the image of the Schur functor $S^{\nu}$ that maps $W$ to its $m$-th tensor power $\otimes^{m} W$ and then 
projects onto the $\nu$-th isotypic component via

$$
w \mapsto \frac{1}{m !} \sum_{\pi \in \mathfrak{S}_{m}} \chi_{\nu}(\pi) \pi w,
$$

where $\chi_{\nu}$ is the character of the irreducible representation of type $\nu$ of the symmetric group $\mathfrak{S}_{m}$. For example, if $\nu=(m)$, then $S^{\nu} W$ is the vector space of symmetric tensors of order $m$, which we denote by $\operatorname{Sym}^{m} W$. If $\nu=(1,1, \ldots, 1)$ is of size $m$, then $S^{\nu} W$ is the vector space of alternating tensors of order $m$, which we denote by $\bigwedge^{m} W$.

A plethysm is the application of $S^{\mu}$ to $S^{\nu} V$. The discussion above shows that this space $S^{\mu} S^{\nu} V:=S^{\mu}\left(S^{\nu} V\right)$ is a also $\mathrm{GL}(V)$ representation. Since $\mathrm{GL}(V)$ is reductive, every finite-dimensional $\mathrm{GL}(V)$-representation decomposes into a direct sum of irreducible $\mathrm{GL}(V)$-representations:

$$
S^{\mu} S^{\nu} V=\bigoplus_{\lambda}\left(S^{\lambda} V\right)^{\oplus p_{\lambda}(\mu, \nu)}
$$

The nonnegative integers $p_{\lambda}(\mu, \nu)$ are called general plethysm coefficients. The case where $\mu=(n)$ and $\nu=(m)$ is of special interest, so we write $a_{\lambda}(n, m):=p_{\lambda}((n),(m))$, which is the multiplicity of type $\lambda$ in $\operatorname{Sym}^{n} \operatorname{Sym}^{m} V$. The coefficient $a_{\lambda}(n, m)$ is often called the plethysm coefficient in the literature. We introduce the dual plethysm coefficient $b_{\lambda}(n, m)$ as another special case of general plethysm coefficients, defined by

$$
\begin{aligned}
\operatorname{Sym}^{n} \operatorname{Sym}^{m} V & =\bigoplus_{\lambda}\left(S^{\lambda} V\right)^{\oplus a_{\lambda}(n, m)}, \\
\bigwedge^{n} \operatorname{Sym}^{m} V & =\bigoplus_{\lambda}\left(S^{\lambda} V\right)^{\oplus b_{\lambda}(n, m)},
\end{aligned}
$$

i.e., by restricting the Young diagrams $\mu$ and $\nu$ to a single row (in case of Sym) or a single column (in case of $\bigwedge$ ). By some well-known dualities (Carr 1990; Manivel 1998; Manivel \& Michałek 2015), we can relate the above decompositions with their respective analogues after exchanging Sym and $\bigwedge$. 
FACT 2.3 (Carr 1990; Manivel 1998; Manivel \& Michałek 2015). If $m$ is odd, then:

$$
\begin{aligned}
\bigwedge^{n} \bigwedge^{m} V & =\bigoplus_{\lambda}\left(S^{\lambda} V\right)^{\oplus a_{\lambda^{t}}(n, m)}, \\
\operatorname{Sym}^{n} \bigwedge^{m} V & =\bigoplus_{\lambda}\left(S^{\lambda} V\right)^{\oplus b_{\lambda^{t}}(n, m)},
\end{aligned}
$$

whereas in case that $m$ is even:

$$
\begin{aligned}
\bigwedge^{n} \bigwedge^{m} V & =\bigoplus_{\lambda}\left(S^{\lambda} V\right)^{\oplus b_{\lambda^{t}}(n, m)}, \\
\operatorname{Sym}^{n} \bigwedge^{m} V & =\bigoplus_{\lambda}\left(S^{\lambda} V\right)^{\oplus a_{\lambda^{t}}(n, m)} .
\end{aligned}
$$

(Notice that here $\lambda$ occurs transposed in $a_{\lambda^{t}}(n, m)$ and $b_{\lambda^{t}}(n, m)$ as opposed to (2.1) and (2.2).)

\section{Main results: complexity of plethysm coefficients}

In order to establish hardness of computing general plethysm coefficients $p_{\lambda}(\mu, \nu)$, it is of course sufficient to focus on the interesting special cases $a_{\lambda}(n, m)$ and $b_{\lambda}(n, m)$. To that end, we study the following problems from a complexity theoretic perspective ${ }^{3}$.

Problem 3.1 (Plethysm). Given a partition $\lambda$ and two integers $n$ and $m$, output $a_{\lambda}(n, m)$.

Problem 3.2 (PlethysmPositivity). Given a partition $\lambda$ and two integers $n$ and $m$, output "accept" if $a_{\lambda}(n, m)>0$, and output "reject" otherwise.

${ }^{3}$ In all problems, the inputs can be encoded in binary or in unary, as it makes no difference for our results below. The hardness results still hold for the unary encoding, while the polynomial-time algorithms still work for the binary encoding. 
Problem 3.3 (DualPlethysm). Given a partition $\lambda$ and two integers $n$ and $m$, output $b_{\lambda}(n, m)$.

Problem 3.4 (DualPlethysmPositivity). Given a partition $\lambda$ and two integers $n$ and $m$, output "accept" if $b_{\lambda}(n, m)>0$, and output "reject" otherwise.

We remark that the computation of $a_{\lambda}(n, 2)$ and $b_{\lambda}(n, 2)$ is possible in polynomial time ${ }^{4}$, so we need to deal with cases $m \geq 3$ only. We will be particularly interested in cases where one of the parameters $m$ or $n$ is fixed. For each of the problems above, we therefore define versions where $m$, respectively, $n$, is fixed by appending $($ INNER $=m)$, respectively $($ OUTER $=n)$, to the problem name. For example, Plethysm(Inner $=3$ ) is the problem "given $\lambda$ and $n$, compute $a_{\lambda}(n, 3)$."

We remark that PlethysmPositivity is called the "zero locus of plethysm coefficients" in Kahle \& Michałek (2016a), where this problem is labeled as "highly nontrivial." Our main result makes this intuitive statement precise. We prove that PLETHYSMPositivity is NP-hard:

Theorem 3.5. PlethysmPositivity $($ inner $=m)$ and DualPlethysmPositivity $($ InNeR $=m$ ) are NP-hard, for any fixed parameter $m \geq 3$. In particular, PlethysmPositivity and DUALPlethysmPositivity are NP-hard.

By a thorough analysis of the proof, we show that PLETHYSM is \#P-hard:

Theorem 3.6. Plethysm $(\operatorname{Inner}=m)$ and DualPlethysm $(\mathrm{INNER}=m)$ are \#P-hard, for any fixed parameter $m \geq 3$. In particular, Plethysm and DualPlethysm are \#P-hard.

${ }^{4}$ By (Stanley 1999a; Weyman 2003, Propositions 2.3.8, 2.3.9), $\mathrm{Sym}^{n} \mathrm{Sym}^{2} V$ decomposes multiplicity-free as $\bigoplus_{\lambda \in A(n)} S^{\lambda} V$, where $A(n)=\{\lambda$ of size $2 n$ : each $\lambda_{i}$ is even $\}$; similarly, it holds that $\bigwedge^{n} \operatorname{Sym}^{2} V=\bigoplus_{\lambda \in B(n)} S^{\lambda} V$, where $B(n)=\left\{\lambda\right.$ of size $2 n$ : if $\lambda_{i} \geq i$, then $\left.\lambda_{i}=\lambda_{i}^{t}+1\right\}$. Testing membership $\lambda \in$ $A(n)$ and $\lambda \in B(n)$ can both be performed in polynomial time in $|\lambda|$. 
Considering Theorem 3.5 and Theorem 3.6, we obtain an interesting complexity-theoretic contrast if we fix the outer parameter instead of the inner, as seen in the following proposition, whose proof can be extracted from Kahle \& Michałek (2016a) ${ }^{5}$.

Proposition 3.7. Plethysm (outer $=n$ ) and DualPlethysm (OUTER $=n$ ) are in $\mathrm{P}$ for any fixed parameter $n$. More generally, computing $p_{\lambda}(\mu,(m))$ can be done in polynomial time if $|\mu|$ is fixed.

This striking difference of complexities between Theorem 3.5 (if $m$ is fixed, then even deciding positivity of $a_{\lambda}(n, m)$ is NP-hard) and Proposition 3.7 (if $n$ is fixed, then even computing the exact value of $a_{\lambda}(n, m)$ can be done in polynomial time) could be interpreted as an explanation why it is considered to be much more difficult to obtain exact results about $a_{\lambda}(n, m)$ for $n \gg m$ compared to the case where $m \gg n$, see e.g., Kahle \& Michałek (2016a, 2018); Kimoto \& Lee (2019); Thibon \& Carré (1992); Weintraub (1990) for evidence.

In addition, the \#P-hardness result leads to a completeness result for the complexity class GapP - that is, the class of all counting problems implementable by a nondeterministic polynomial-time Turing machine, where the output is interpreted as the number accepting computation paths minus the number of rejecting computation paths (Fenner et al. 1994).

Theorem 3.8. Plethysm, DualPlethysm and the problem of computing general plethysm coefficients are GapP-complete.

The proof of Theorem 3.8 is provided in Section 9.

\section{Fixing the inner parameter to $m=3$}

To ultimately prove Theorem 3.5 and Theorem 3.6, we first demonstrate that proving hardness of the case $m=3$ is enough:

\footnotetext{
${ }^{5}$ Kahle \& Michałek (2016a) shows that, for any fixed partition $\mu$, the function $\left(\lambda_{0}, \ldots, \lambda_{|\mu|-1}\right) \mapsto p_{\lambda}(\mu,(m))$ has a constant size arithmetic formula (with modular arithmetic) whose inputs are the $\lambda_{i}$; see the appendix of the arXiv version (Kahle \& Michałek 2016b) for a good exposition.
} 
Lemma 4.1. Let $m$ be fixed. Given a partition $\lambda$ encoded in unary, we can compute partitions $\pi$ and $\pi^{\prime}$ in polynomial time so that $a_{\lambda}(n, m)=b_{\pi}(n, m+1)$ and $b_{\lambda}(n, m)=a_{\pi^{\prime}}(n, m+1)$.

In particular, provided that deciding positivity of both $a_{\lambda}(n, 3)$ and $b_{\lambda}(n, 3)$ is already NP-hard, deciding positivity of a plethysm coefficient $a_{\lambda}(n, m)$ or $b_{\lambda}(n, m)$ is NP-hard as well for each fixed $m \geq 3$. In order to prove Lemma 4.1, we leverage another fact:

FACT 4.2 (Manivel \& Michałek 2015). Let $\nu$ be a partition of $n$, let $\lambda$ be a partition of $n m$ of width at most $n$ and let $\pi$ be the partition after prepending a row of width $n$ to $\lambda$. Then the multiplicity of the irreducible component $S^{\lambda} V$ in $S^{\nu} \wedge^{m} V$ equals the multiplicity of the irreducible component $S^{\pi} V$ in $S^{\nu} \bigwedge^{m+1} V$.

Proof of Lemma 4.1. We show the first claim only; the second part is analogous. Let a partition $\lambda$ of $n m$ be given. Fact 2.3 yields that $a_{\lambda}(n, m)$ equals the multiplicity of the irreducible representation $S^{\lambda^{t}} V$ in the decomposition of $S^{\nu} \bigwedge^{m} V$, where $\nu$ is the Young Diagram consisting of a single row of width $n$ if $m$ is even and $\nu$ equals a column of length $n$ otherwise. If $\lambda^{t}$ is of width $>n$, then we immediately deduce that $a_{\lambda}(n, m)=0$. So assume that $\lambda^{t}$ is of width $\leq n$ and construct a partition $\pi^{t}$ by prepending a row of width $n$ to $\lambda^{t}$. From Fact 4.2, we infer that the multiplicity of $S^{\pi^{t}}$ in the decomposition of $S^{\nu} \bigwedge^{m+1}$ equals $a_{\lambda}(n, m)$ and by applying Fact 2.3 once more, we finally obtain that $a_{\lambda}(n, m)=b_{\pi}(n, m+1)$.

We are left to deal with the case $m=3$.

\section{Combinatorial descriptions for plethysm coefficients via discrete tomography}

Our results in Section 3 are based on a new combinatorial description of the plethysm coefficient in a subcase, based on new combinatorial lower and upper bounds for the plethysm coefficient. We think that the lower and upper bound as well as the combinatorial description are of independent interest. 
By $[i, j]$, we denote the range $\{i, \ldots, j\} \subseteq \mathbb{N}$. We define $C:=$ $\{(x, y, z): x>y>z\} \subseteq \mathbb{N}^{3}$ as the open cone and $\bar{C}=\{(x, y, z)$ : $x \geq y \geq z\} \subseteq \mathbb{N}^{3}$ as the closed cone. A finite set $P \subseteq C$ is called a point set in the open cone. A finite set $P \subseteq \bar{C}$ is called a point set in the closed cone. A point set $P$ in the open cone is called a pyramid in the open cone if for all $(x, y, z) \in P$ and all $\left(x^{\prime}, y^{\prime}, z^{\prime}\right) \in C$ with $x^{\prime} \leq x, y^{\prime} \leq y, z^{\prime} \leq z$ we have $\left(x^{\prime}, y^{\prime}, z^{\prime}\right) \in P$. Analogously, a point set $P$ in the closed cone is called a pyramid in the closed cone if for all $(x, y, z) \in P$ and all $\left(x^{\prime}, y^{\prime}, z^{\prime}\right) \in \bar{C}$ with $x^{\prime} \leq x, y^{\prime} \leq y, z^{\prime} \leq z$ we have $\left(x^{\prime}, y^{\prime}, z^{\prime}\right) \in P$.

Let $P$ be a point set in the open cone or closed cone. The sum-marginal $S(P)$ is the sequence of numbers defined via

$$
S_{i}(P):=\sum_{(x, y, z) \in P} \delta_{x, i}+\delta_{y, i}+\delta_{z, i},
$$

where $\delta_{i, j}=1$ if $i=j$ and $\delta_{i, j}=0$ otherwise. Note that $|S(P)|:=$ $\sum_{i} S_{i}(P)=3|P|$.

Definition 5.2. Let $\lambda$ be a partition of $3 n$. We define:

- $a_{\lambda}^{-}(n, 3)$ as the number of pyramids in the open cone with sum-marginal $\lambda^{t}$,

- $a_{\lambda}^{+}(n, 3)$ as the number of point sets in the open cone with sum-marginal $\lambda^{t}$,

- $b_{\lambda}^{-}(n, 3)$ as the number of pyramids in the closed cone with sum-marginal $\lambda$,

- $b_{\lambda}^{+}(n, 3)$ as the number of point sets in the closed cone with sum-marginal $\lambda$.

The next theorem connects plethysm coefficients to the combinatorial notions that we just defined.

TheOREM 5.3. Let $\lambda$ be a partition of $3 n$. Then:

$$
a_{\lambda}^{-}(n, 3) \leq a_{\lambda}(n, 3) \leq a_{\lambda}^{+}(n, 3), b_{\lambda}^{-}(n, 3) \leq b_{\lambda}(n, 3) \leq b_{\lambda}^{+}(n, 3) .
$$


Proof. Let $X_{0}, X_{1}, \ldots, X_{k-1}$ be an ordered basis of $V=\mathbb{C}^{k}$. There is a natural bijection between the points in $\bar{C}_{<k}:=[0, k-$ $1]^{3} \cap \bar{C}$ and the monomials in $\operatorname{Sym}^{3} V$. It is easy to check that a monomial $X_{x} X_{y} X_{z}$ is of weight $\kappa$ if and only the corresponding point $(x, y, z)$ has sum-marginal $\kappa$ (here, $\kappa$ is not necessarily a partition). In the same way, we match $(x, y, z) \in C_{<k}:=[0, k-$ $1]^{3} \cap C$ with elementary wedge products $X_{x} \wedge X_{y} \wedge X_{z}$. (Since we are dealing with these objects modulo scalars, the sign changes caused by the wedge product shall not bother us here.)

Now let $\lambda$ be a partition of $3 n$. We associate each point set $P \subseteq \bar{C}_{<k}$ with sum-marginal $\lambda$ with a vector

$$
v_{P}:=\bigwedge_{(x, y, z) \in P} X_{x} X_{y} X_{z} \in \bigwedge^{n} \operatorname{Sym}^{3} V ;
$$

notice that $|\lambda|=3 n$ implies that $P$ is of size $n$ and thus indeed $v_{P} \in \wedge^{n} \operatorname{Sym}^{3} V$. Analogously, for point sets $P \subseteq C_{<k}$ with summarginal $\lambda$, we define vectors

$$
w_{P}:=\bigwedge_{(x, y, z) \in P} X_{x} \wedge X_{y} \wedge X_{z} \in \bigwedge^{n} \bigwedge^{3} V .
$$

From the preceding paragraphs, it follows that $v_{P}$ is a weight vector of weight $\lambda$, and furthermore, for $P$ ranging over all point subsets of $\bar{C}_{<k}$ with sum-marginal $\lambda,\left\{v_{P}\right\}_{P}$ forms a basis of the weight subspace of weight $\lambda$ of $\bigwedge^{n} \operatorname{Sym}^{3} V$. Recall that $b_{\lambda}^{+}(n, 3)$ exactly counts the number of such sets $P$, thus $b_{\lambda}^{+}(n, 3)$ equals the dimension of the weight- $\lambda$ subspace. Moreover, since $\Lambda^{n} \mathrm{Sym}^{3} V$ is a $\mathrm{GL}(V)$-representation, the multiplicity $b_{\lambda}(n, 3)$ of $\lambda$ in the decomposition of $\bigwedge^{n} \mathrm{Sym}^{3} V$ into irreducible $\mathrm{GL}(V)$-representations matches the dimension of the linear subspace spanned by all highest weight vectors of weight $\lambda$. But each highest weight vector is in particular a weight vector and thus $b_{\lambda}(n, 3) \leq b_{\lambda}^{+}(n, 3)$.

In the same way, $\left\{w_{P}\right\}_{P}$ forms a basis of the weight- $\lambda^{t}$ subspace of $\Lambda^{n} \Lambda^{3} V$, for $P$ ranging over all point subsets of $C_{<k}$ with sum-marginal $\lambda^{t}$. Therefore, $a_{\lambda}^{+}(n, 3)$ equals the dimension of the weight- $\lambda^{t}$ subspace and hence upper-bounds the multiplicity of the irreducible component $S^{\lambda^{t}} V$ in $\Lambda^{n} \Lambda^{3} V$. Recall that this multiplicity equals $a_{\lambda}(n, 3)$ by Fact 2.3, so we conclude $a_{\lambda}(n, 3) \leq a_{\lambda}^{+}(n, 3)$. 
For the remainder of the proof, we can treat the symmetric (closed cone) and skew-symmetric (open cone) cases identically, so we omit the proof of $a_{\lambda}^{-}(n, 3) \leq a_{\lambda}(n, 3)$ and verify only that $b_{\lambda}^{-}(n, 3) \leq b_{\lambda}(n, 3)$. Let $P \subseteq \bar{C}_{<k}$ be a pyramid in the closed cone with sum-marginal $\lambda,|\lambda|=3 n$. It suffices to show that $v_{P}$ is not only a weight vector, but in fact a highest weight vector. To prove this, we demonstrate that $v_{P}$ is annihilated by all raising operators $E_{i, j}$; recall that, for $i<j, E_{i, j}$ denotes the upper triangular matrix with a single 1 in the $i$-th row and the $j$-th column and zero entries anywhere else.

$$
\begin{aligned}
E_{i, j} v_{P}= & \lim _{\varepsilon \rightarrow 0} \varepsilon^{-1}\left(\left(\mathrm{Id}+\varepsilon E_{i, j}\right) v_{P}-v_{P}\right) \\
=\sum_{(x, y, z) \in P}\left(\delta_{x, j} X_{i} X_{y} X_{z}+\delta_{y, j} X_{x} X_{i} X_{z}+\delta_{z, j} X_{x} X_{y} X_{i}\right) & \wedge \bigwedge_{\substack{\left.x^{\prime}, y^{\prime}, z^{\prime}\right) \in \\
P \backslash\{(x, y, z)\}}} X_{x^{\prime}} X_{y^{\prime}} X_{z^{\prime}} .
\end{aligned}
$$

We claim that each summand vanishes individually. Focus on any summand $\delta_{x, j} X_{i} X_{y} X_{z} \wedge \bigwedge_{\left(x^{\prime}, y^{\prime}, z^{\prime}\right)} X_{x^{\prime}} X_{y^{\prime}} X_{z^{\prime}}$, where $(x, y, z) \in P$ and $\left(x^{\prime}, y^{\prime}, z^{\prime}\right) \in P \backslash\{(x, y, z)\}$. In case that $x \neq j$, this term clearly equals zero, so assume $x=j$. Since $i<j=x$, the points $(i, y, z)$ and $(x, y, z)$ differ. But $P$ is a pyramid and thus contains $(i, y, z)$, too. Consequently, the monomial $X_{i} X_{y} X_{z}$ also occurs in the big wedge product on the right-hand side and therefore cancels with $X_{i} X_{y} X_{z}$ on the left-hand side. The other summands with $i$ in place of $y$ or $z$, respectively, can be shown to vanish by an analogous argumentation.

Applying the raising operator $E_{i, j}$ to $v_{P}$ has an intuitive geometric interpretation for $P$ : Any summand as above arises from $P$ by replacing some coordinate $j$ of some point in $P$ by $i$. Thereby that point is moved closer to the origin as $i<j$. Recall, however, that pyramids are point sets not containing "holes," thus $P$ is transformed by mapping two points onto eah other. Here comes the alternating property of the wedge product into play by annihilating the summand.

When the lower and upper bound coincide, we get a combinatorial description of the (dual) plethysm coefficient. The following 
Proposition 5.5 gives a sufficient condition for when this happens. Let $\lambda \in \mathbb{N}^{[0, r]}$ be a composition. We define the coordinate sum $B(\lambda)$ via

$$
B(\lambda)=\sum_{i=0}^{r} i \cdot \lambda_{i}
$$

Let $P$ be a point set in the open cone or closed cone. The coordinate sum $B(P)$ is defined as the coordinate sum of the summarginal $B(P):=B(S(P))$, which is the same as the sum over all coordinates:

$$
B(P)=\sum_{(x, y, z) \in P} x+y+z
$$

Let $\bar{\xi}(i)$ the number of cardinality-1 point sets in the closed cone with coordinate sum exactly $i$. This is the same as the number of partitions of $i$ of height $\leq 3$, which is OEIS sequence A001399, with explicit formula round $\left((i+3)^{2} / 12\right)$. Let $\xi(i)$ denote the number of cardinality-1 point sets in the open cone with coordinate sum $i$. This is the same as the number of partitions $\mu$ of $i$ of height 3 such that $\mu_{1}, \mu_{2}$, and $\mu_{3}$ are pairwise distinct, which is OEIS sequence A069905 ${ }^{6}$, with explicit formula round $\left(i^{2} / 12\right)$.

Now let $\bar{\iota}(n):=\min \left\{\iota: \sum_{i=0}^{\iota} \bar{\xi}(i) \geq n\right\}$ and analogously, let $\iota(n):=\min \left\{\iota: \sum_{i=0}^{\iota} \xi(i) \geq n\right\}$. Finally, let $\bar{\beta}(n):=\sum_{i=1}^{n} \bar{\iota}(i)$ and analogously, let $\beta(n):=\sum_{i=1}^{n} \iota(i)$.

Lemma 5.4. There is no point set $P$ of cardinality $n$ in the closed cone that has $B(P)<\bar{\beta}(n)$. Analogously, there is no point set $P$ of cardinality $n$ in the open cone that has $B(P)<\beta(n)$.

Proof. This is seen by induction: If $P$ of cardinality $n$ in the open cone has $B(P)<\beta(n)$, then remove the point $(x, y, z)$ with highest coordinate sum to obtain the point set $P^{\prime}$. Since for each $\iota$, there are only $\xi(\iota)$ many points $\left(x^{\prime}, y^{\prime}, z^{\prime}\right)$ in $C$ with $x^{\prime}+y^{\prime}+z^{\prime}=\iota$, it follows that $x+y+z \geq \iota(n)$. But $\beta(n)-\iota(n)=\beta(n-1)$,

${ }^{6}$ There is a bijection to partitions with 3 positive but not necessarily distinct parts via subtracting $(2,1,0)$. 
which implies $B\left(P^{\prime}\right) \leq B(P)-\iota(n)<\beta(n)-\iota(n)=\beta(n-1)$, which means that $P^{\prime}$ cannot exist by induction hypothesis and hence $P$ cannot exist. A completely analogous argument works for the closed cone.

Proposition 5.5. If $B(\lambda)=\bar{\beta}(|\lambda| / 3)$, then each point set in the closed cone with sum-marginal $\lambda$ is a pyramid in the closed cone. In particular, if $\lambda$ is a partition of $3 n$, according to Theorem 5.3, we have $b_{\lambda}^{-}(n, 3)=b_{\lambda}(n, 3)=b_{\lambda}^{+}(n, 3)$.

If $B\left(\lambda^{t}\right)=\beta(|\lambda| / 3)$, then each point set in the open cone with sum-marginal $\lambda^{t}$ is a pyramid in the open cone. In particular, if $\lambda$ is a partition of $3 n$, then according to Theorem 5.3, we have $a_{\lambda}^{-}(n, 3)=a_{\lambda}(n, 3)=a_{\lambda}^{+}(n, 3)$.

Proof. Assume for the sake of contradiction that there exists a point set $P$ in the closed cone with sum-marginal $S(P)=\lambda$ (note that $S(P)=\lambda$ implies $|P|=|\lambda| / 3$ ) such that $P$ is not a pyramid in the closed cone. Then there exists $(x, y, z) \in P$ and $\left(x^{\prime}, y^{\prime}, z^{\prime}\right) \notin P$ with $x^{\prime} \leq x, y^{\prime} \leq y, z^{\prime} \leq z$. Replacing $(x, y, z)$ in $P$ with $\left(x^{\prime}, y^{\prime}, z^{\prime}\right)$ gives a point set $P^{\prime}$ with $B\left(P^{\prime}\right)<B(P)$. This is a contradiction to Lemma 5.4. A completely analogous argument works for the open cone.

\section{Proof of main theorems 3.5 and 3.6}

The reductions that we present are parsimonious polynomial-time reductions between counting problems. Such reductions automatically yield polynomial-time many-one reductions between the associated decision problems.

\subsection{Counting and decision problems in discrete tomogra-} phy. Let us start with some notations: Let $P \subseteq \mathbb{N}^{3}$ be a finite set of points. By $X_{i}(P)$ we denote the number of elements in $P$ with $X$-coordinate $i$. Similarly, $Y_{i}(P)$ and $Z_{i}(P)$ count the elements with $Y$ - and $Z$-coordinate $i$, respectively. We call $X(P):=$ $\left(X_{i}(P)\right)_{i}, Y(P):=\left(Y_{i}(P)\right)_{i}$ and $Z(P):=\left(Z_{i}(P)\right)_{i}$ the $X$-, $Y$ - and $Z$-marginals of $P$; these sequences are treated as finite by omitting 

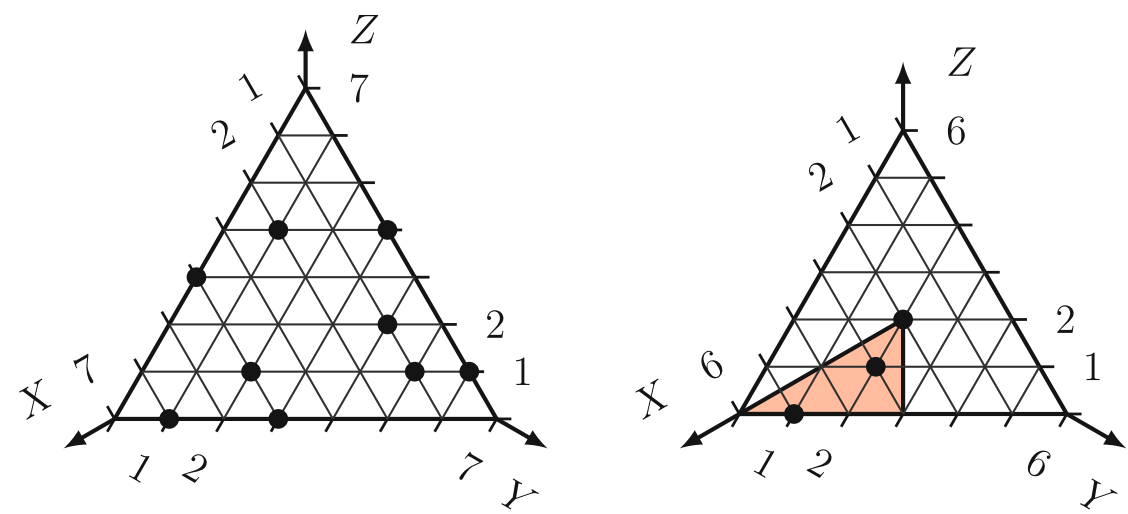

Figure 6.1: The left depiction of $P \subseteq G_{7}$ certifies that the 2D-X-RAY instance defined by $\mu=(2,2,1,0,3,0,1,0), \nu=$ $(1,2,1,2,1,1,1,0)$ and $\rho=(2,3,1,1,2,0,0,0)$ is satisfiable. The right image gives an example of a set $P \subseteq G_{6} \cap \bar{C}$ with sum-marginal $\lambda=(0,0,1,1,0,1,0)+(0,1,2,0,0,0,0)+$ $(1,1,1,0,0,0,0)=(1,2,4,1,0,1,0)$. The framed area (including the boundary) illustrates the closed cone $\bar{C}$, while the interior of that area illustrates the open cone $C$.

trailing zeros. Recall the definition (5.1) of the sum-marginal $S(P)$ and observe that $S(P)=X(P)+Y(P)+Z(P)$.

The intermediate 2-dimensional problems below operate on the grid

$$
G_{r}:=\{(x, y, z): x+y+z=r\} \subseteq \mathbb{N}^{3} ;
$$

in spirit, $G_{r}$ is two-dimensional although each entry of $G_{r}$ is parameterized by 3 coordinates (the so-called trilinear coordinates). This specific embedding of $G_{r} \subseteq \mathbb{N}^{3}$ plays a major role in the proof of Lemma 6.9.

Problem 6.1. [2D-X-RAY] Given $\mu, \nu, \rho \in \mathbb{N}^{[0, r]}$, the 2D-X-RAY problem is to decide if there exists a point set $P \subseteq G_{r}$ with $X-, Y$ and $Z$-marginals $\mu, \nu$ and $\rho$, respectively.

Figure 6.1 exemplifies an instance of 2D-X-RAY with $r=7$. For this problem and all following tomography problems, we denote the respective counting versions by prepending \#. For example: 


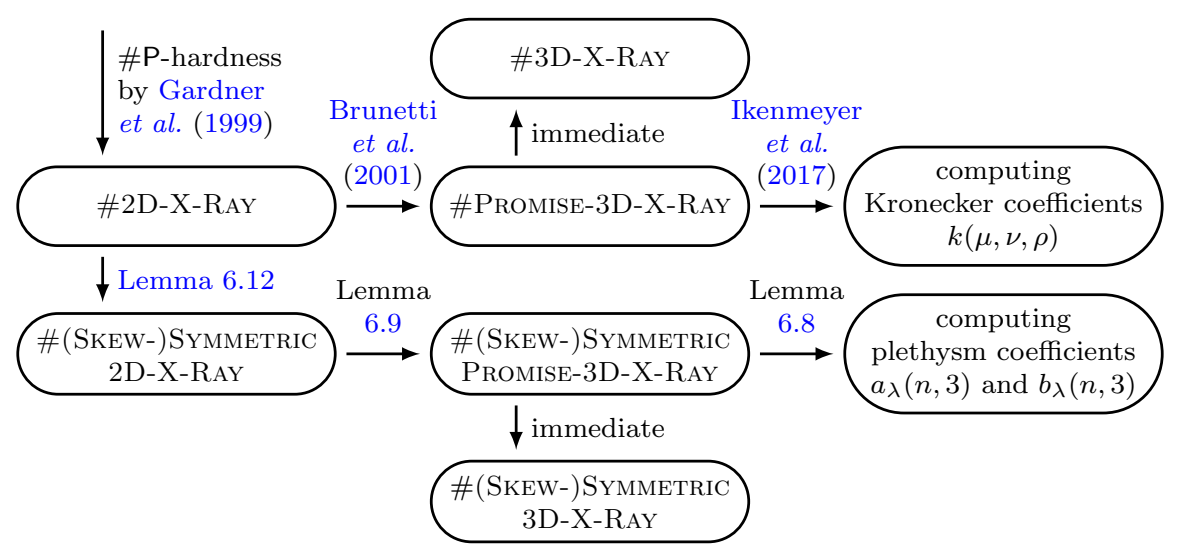

Figure 6.2: The reductions that lead to \#P-hardness proofs for the problems of computing Kronecker and plethysm coefficients. All depicted reductions are parsimonious and polynomial-time, so the NP-hardness of all corresponding decision problems ultimately follows from the NP-hardness of 2D-X-RAY that was proved in Gardner et al. (1999).

Problem 6.2 (\#2D-X-RAy). Given $\mu, \nu, \rho \in \mathbb{N}^{[0, r]}$, the \#2D$\mathrm{X}$-RAY problem is to compute the number of point sets $P \subseteq G_{r}$ with $X-, Y$ - and $Z$-marginals $\mu, \nu$ and $\rho$, respectively.

2D-X-RAY has been identified as NP-hard (even NP-complete) before by Gardner, Gritzmann, and Prangenberg Gardner et al. (1999). In fact, they prove that the counting version \#2D-XRAY is \#P-hard (even \#P-complete). Keeping this in mind, we now continue to reduce \#2D-X-RAY in a parsimonious way to computing $a_{\lambda}(n, 3)$ or $b_{\lambda}(n, 3)$, taking the detour over the other \#X-RAY problems. Our reductions are illustrated in Figure 6.2.

Problem 6.3. (Symmetric 2D-X-RAy and Skew-Symmetric 2D-X-RAY) Given $\lambda \in \mathbb{N}^{[0, r]}$, the Symmetric 2D-X-RAY problem is to decide if there exists a point set $P \subseteq G_{r} \cap \bar{C}$ with summarginal $S(P)=\lambda$.

Analogously, given $\lambda \in \mathbb{N}^{[0, r]}$, the SkEw-Symmetric 2D-XRAY problem is to decide if there exists a point set $P \subseteq G_{r} \cap C$. 
Finally, we introduce three-dimensional variants that differ from the above problems only in that $P$ is taken from $\mathbb{N}^{3}$ rather than $G_{r}$ :

Problem 6.4 (3D-X-RAY). Given $\mu, \nu, \rho \in \mathbb{N}^{[0, r]}$, the 3D-XRAY problem is to decide if there exists a point set $P \subseteq \mathbb{N}^{3}$ with $X$-, $Y$ - and $Z$-marginals $\mu, \nu$ and $\rho$, respectively.

3D-X-RAY is known to be NP-complete by Brunetti et al. (2001), see Figure 6.2.

Problem 6.5. (Symmetric 3D-X-RAy and Skew-Symmetric 3D-X-RAY) Given $\lambda \in \mathbb{N}^{[0, r]}$, the Symmetric 3D-X-RAY problem is to decide if there exists a point set $P \subseteq \bar{C}$ with summarginal $\lambda$.

Analogously, given $\lambda \in \mathbb{N}^{[0, r]}$, the Skew-Symmetric 3D-X$\mathrm{RAY}$ problem is to decide if there exists a point set $P \subseteq C$ with sum-marginal $\lambda$.

Our main focus in 3D will be the following promise problem version.

Problem 6.6. (Symmetric Promise-3D-X-Ray and SkewSymmetricPromise-3D-X-RAY) Given $\lambda \in \mathbb{N}^{[0, r]}$ such that $B(\lambda)=\bar{\beta}(|\lambda| / 3)$, the Symmetric Promise-3D-X-RAY problem is to decide if there exists a point set $P \subseteq \bar{C}$ with sum-marginal $\lambda$.

Analogously, given $\lambda \in \mathbb{N}^{[0, r]}$ such that $B(\lambda)=\beta(|\lambda| / 3)$, the Skew-Symmetric Promise-3D-X-RAY problem is to decide if there exists a point set $P \subseteq C$ with sum-marginal $\lambda$.

6.2. Reduction from \#(Skew-)Symmetric Promise-3D-XRAY to (DUAL-)Plethysm. Recall that instances $\lambda$ of (SKEW-) Symmetric Promise-3D-X-RAY need not be partitions in general. Still, we prove that any promise instance $\lambda$ which is not a partition is trivially rejected:

LEMMA 6.7. Let $\lambda$ be a composition and assume there exists some pyramid $P$ in the open or closed cone with sum-marginal $\lambda$. Then $\lambda$ is a partition. 
Proof. The proof is slightly subtle. Fix an arbitrary $i$; we want to show that $\lambda_{i} \geq \lambda_{i+1}$. Let us define $\alpha(x, y, z):=\delta_{x, i}+\delta_{y, i}+$ $\delta_{z, i}-\delta_{x, i+1}-\delta_{y, i+1}-\delta_{z, i+1}$. It is easy to see that $\lambda_{i}-\lambda_{i+1}=$ $\sum_{(x, y, z) \in P} \alpha(x, y, z)$, so we have to show that $\sum_{(x, y, z) \in P} \alpha(x, y, z) \geq$ 0 . We achieve this as follows: To each point $(x, y, z) \in P$ with $\alpha(x, y, z)<0$, we assign a point $\left(x^{\prime}, y^{\prime}, z^{\prime}\right) \in P$ such that $\alpha(x, y, z)+$ $\alpha\left(x^{\prime}, y^{\prime}, z^{\prime}\right)=0$. Every $\left(x^{\prime}, y^{\prime}, z^{\prime}\right)$ will be used for at most one $(x, y, z)$, which then finishes the proof. We proceed to define $\left(x^{\prime}, y^{\prime}, z^{\prime}\right)=: \varphi(x, y, z)$. The symbol $\cdot$ matches any input $\notin\{i, i-1\}$ and is not modified in the output. We set $\varphi(i+1, i+1, i+1):=$ $(i, i, i), \varphi(i+1, i+1, i):=(i+1, i, i), \varphi(i+1, i+1, \cdot):=(i, i, \cdot)$, $\varphi(\cdot, i+1, i+1):=(\cdot, i, i), \varphi(i+1, \cdot, \cdot):=(i, \cdot, \cdot), \varphi(\cdot, i+1, \cdot):=$ $(\cdot, i, \cdot), \varphi(\cdot, \cdot, i+1):=(\cdot, \cdot, i)$. The map $\varphi$ maps points in $C$ to points in $C$, and points in $\bar{C}$ to points in $\bar{C}$. Moreover, since $P$ is a pyramid, we have indeed $\left(x^{\prime}, y^{\prime}, z^{\prime}\right) \in P$.

Lemma 6.8. There is a parsimonious polynomial-time reduction from \#Symmetric Promise-3D-X-Ray to DualPlethysm $($ INNER $=3)$.

Moreover, there is a parsimonious polynomial-time reduction from \#Skew-Symmetric Promise-3D-X-Ray to Plethysm $($ INNER $=3)$.

Proof. We make use of a trivial no-instance for DuALPlethysm: $b_{(2,1)}(1,3)=0$. Given an instance $(\lambda, n)$ of \#SYMMETRIC PRomise-3D-X-RAY, if $\lambda$ is not a partition or if $|\lambda| \neq 3 n$, then the reduction outputs that trivial no-instance. This is the correct behavior according to Lemma 6.7.

On the other hand, if $\lambda$ is a partition, then the reduction outputs its input $(\lambda, n)$. Since $B(\lambda)=\bar{\beta}(|\lambda| / 3)$, Proposition 5.5 ensures that $b_{\lambda}^{+}(n, 3)=b_{\lambda}(n, 3)$. Observe that in the language of Section 5, \#Symmetric Promise-3D-X-Ray asks to compute $b_{\lambda}^{+}(n, 3)$. Hence the reduction works correctly.

The same argument works for $a_{\lambda}(n, 3)$ and $\beta(|\lambda| / 3)$. Here, in the nontrivial case, $\left(\lambda^{t}, n\right)$ is returned.

6.3. Reduction from \#(SkEW-)SYMmETRIC 2D-X-RAY to \# (Skew-)Symmetric Promise-3D-X-Ray. In this section, 
we prove the following lemma.

LEMMA 6.9. There is a parsimonious polynomial-time reduction from \#Symmetric 2D-X-RAY to \#Symmetric Promise-3D$\mathrm{X}-\mathrm{RAY}$.

Moreover, there is a parsimonious polynomial-time reduction from \#SKew-Symmetric 2D-X-RAY to \#SKEW-Symmetric PROMISE-3D-X-RAY.

Before we can prove Lemma 6.9, we make an observation (see Lemma 6.10 below). We call $P_{r}:=\{(x, y, z): x+y+z \leq r, x>$ $y>z\}$ the complete pyramid in the open cone, and analogously $\overline{P_{r}}:=\{(x, y, z): x+y+z \leq r, x \geq y \geq z\}$ the complete pyramid in the closed cone. Note that for all $r$ we have $\bar{\beta}\left(\left|\bar{P}_{r}\right|\right)=B\left(\bar{P}_{r}\right)$. Moreover, $\bar{\beta}(n)$ is the piecewise linear interpolation between these values. Analogously, $\beta(n)$ is the piecewise linear interpolation between $\beta\left(\left|P_{r}\right|\right)=B\left(P_{r}\right)$.

The proof of the following lemma is a careful adaption of a proof given by Brunetti, Del Lungo and Grard Brunetti et al. (2001) for the nonsymmetric 3D-X-RAY problem. Roughly speaking, the trick is to embed the two-dimensional grid $G_{r}$ into the $r$-th layer of the complete pyramid.

Lemma 6.10. Let $P \subseteq \bar{C}$ be of size $|P|=n$. Then $P$ has coordinate sum $B(P)=\bar{\beta}(n)$ if and only if $\bar{P}_{r-1} \subseteq P \subseteq \bar{P}_{r}$ for some $r$.

Analogously, let $P \subseteq C$ be of size $|P|=n$. Then $P$ has coordinate sum $B(P)=\beta(n)$ if and only if $P_{r-1} \subseteq P \subseteq P_{r}$ for some $r$.

Proof. It is easy to see by a direct calculation that all point sets $\bar{P}_{r-1} \subseteq P \subseteq \bar{P}_{r}$ have $B(P)=\bar{\beta}(n)$, since every point in $P \backslash \bar{P}_{r-1}$ has the same coordinate sum $r$ (i.e., $P \backslash \bar{P}_{r-1} \subseteq G_{r}$ ).

The other direction is intuitively straightforward by the following argument: Whenever $P$ contains a "hole," then we can fill the hole by moving outer points closer to the origin. But in that we decrease the coordinate sum of $P$. More formally: Assume that $P$ has minimal coordinate sum among all sets $\subseteq \bar{C}$ of the same size $n$. We pick $r$ to be maximal, such that $\bar{P}_{r-1} \subseteq P$ and assume, for the sake of contradiction, that $P \nsubseteq \bar{P}_{r}$, i.e., there exists some $(x, y, z) \in P \backslash \bar{P}_{r}$. Furthermore, there exists some $\left(x^{\prime}, y^{\prime}, z^{\prime}\right) \in$ 

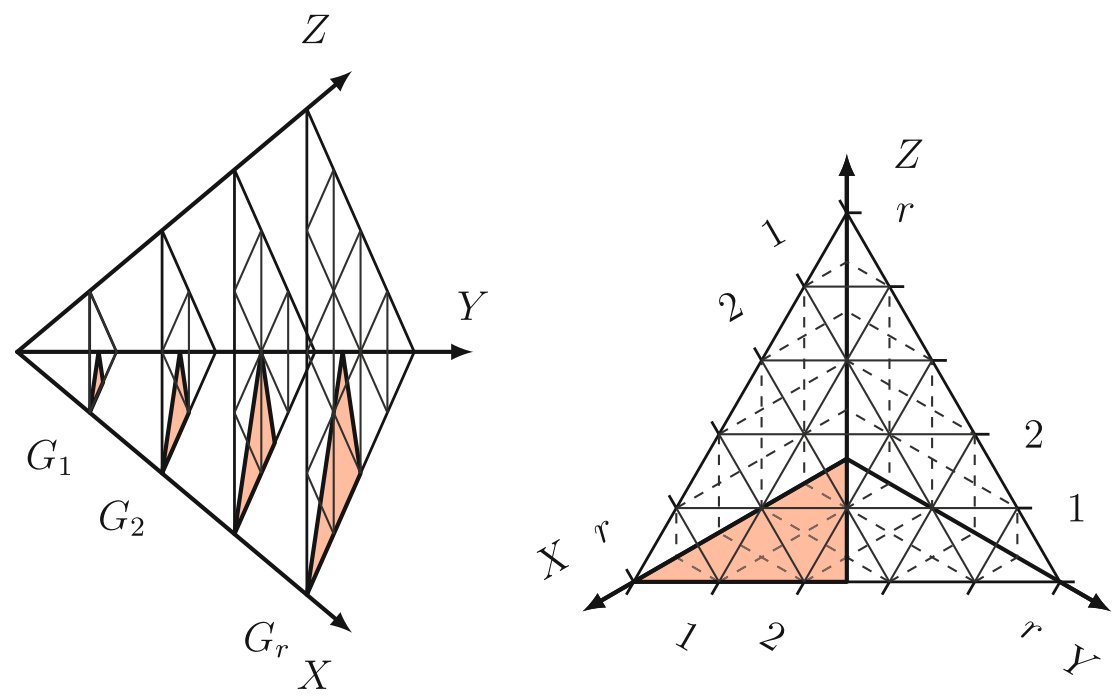

Figure 6.3: Visualizes how to arrange the grids $G_{r}$ in threedimensional space; the red-colored areas indicate the intersections with $\bar{C}$ (or $C$, by restricting to the interior). The essential step of the reduction is to embed a set $\widehat{P} \subseteq G_{r}$ into the top-most layer of the pyramid $P:=P_{r-1} \cup \widehat{P}$.

$\bar{P}_{r} \backslash P$, since otherwise $P \subseteq \bar{P}_{r}$ which contradicts the way we chose $r$. We construct the set $P^{\prime}:=P \cup\left\{\left(x^{\prime}, y^{\prime}, z^{\prime}\right)\right\} \backslash\{(x, y, z)\}$; clearly $\left|P^{\prime}\right|=|P|=n$. The coordinate sum of $P^{\prime}$ is bounded by

$$
B\left(P^{\prime}\right)=B(P)-\underbrace{(x+y+z)}_{>r}+\underbrace{\left(x^{\prime}+y^{\prime}+z^{\prime}\right)}_{\leq r}<B(P)=\bar{\beta}(n),
$$

which is a contradiction to the definition of $\bar{\beta}(n)$.

This proof works completely analogously for the open cone.

The reduction now proceeds by embedding $G_{r}$ in the threedimensional space. Any solution $\widehat{P}$ of the given (SkEW-)SYMMETRIC 2D-X-RAY instance is interpreted as the $r$-th layer of the thereunder entirely filled pyramid $P=\bar{P}_{r-1} \cup \widehat{P}$; Figure 6.3 might give some further intuition on how to view Symmetric 2D-XRAY in three-dimensional space. We detail the construction in the following lemma: 
Lemma 6.11. Fix some $r$. Let $\widehat{\lambda} \in \mathbb{N}^{[0, r]}$ be such that there exists $n$ with $|\widehat{\lambda}|=3 n$ and $B(\widehat{\lambda})=r n$. (i.e., $\widehat{\lambda}$ is not trivially rejected as a Symmetric 2D-X-RAY instance). Let $\lambda:=S\left(\bar{P}_{r-1}\right)+\widehat{\lambda}$. Then $B(\lambda)=\bar{\beta}\left(\left|\bar{P}_{r-1}\right|+n\right)$ and there is a one-to-one correspondence between point sets $P \subseteq \bar{C}$ with sum-marginal $\lambda$ and point sets $\widehat{P} \subseteq G_{r} \cap \bar{C}$ with sum-marginal $\widehat{\lambda}$.

The same statements hold if instead we consider point sets in the open cone $C, P_{r-1}$ and $P_{r}$, and the Skew-Symmetric problems. In that case $\lambda:=S\left(P_{r-1}\right)+\widehat{\lambda}$.

Proof. We calculate $B(\lambda)=B\left(\bar{P}_{r-1}\right)+r n=\bar{\beta}\left(\left|\bar{P}_{r-1}\right|\right)+r n=$ $\bar{\beta}\left(\left|\bar{P}_{r-1}\right|+n\right)$. The claimed one-to-one correspondence works as follows: For $P \subseteq \bar{C}$ with sum-marginal $\lambda=S\left(\bar{P}_{r-1}\right)+\widehat{\lambda}$ we know (Lemma 6.10) that $P=\bar{P}_{r-1} \cup \widehat{P}$ for some $\widehat{P} \subseteq \bar{C} \cap G_{r}$. The set $\widehat{P}$ has sum-marginal $\hat{\lambda}$. Conversely, if some set $\widehat{\widehat{P}}$ has sum-marginal $\widehat{\lambda}$, then the set $P:=\bar{P}_{r-1} \cup \widehat{P}$ has sum-marginal $S\left(\bar{P}_{r-1}\right)+\widehat{\lambda}=\lambda$. Both maps $P \mapsto \widehat{P}$ and $\widehat{P} \mapsto P$ are inverse to each other, which finishes the proof.

After applying the obvious changes, the proof also works for the open cone $C$.

Proof of Lemma 6.9. Given a \#Symmetric 2D-X-Ray instance $\widehat{\lambda}$, we first assert that $|\widehat{\lambda}|$ is divisible by 3 and that $B\left(\widehat{\lambda}_{i}\right)=$ $r n$ holds for $n=|\widehat{\lambda}| / 3$. If this step fails, output a trivially zero \#Symmetric 3D-X-RAY instance. Otherwise, construct $\lambda:=$ $S\left(\bar{P}_{r-1}\right)+\widehat{\lambda}$ and output $\lambda$ as the corresponding \#SYMmETRIC 3D-X-RAY instance. Clearly, this algorithm runs in polynomial time.

Lemma 6.11 entirely proves the remaining goals: Since $B(\lambda)=$ $\bar{\beta}\left(\left|\bar{P}_{r-1}\right|+n\right)$, the promise is kept. Moreover, the one-to-one correspondence in Lemma 6.11 ensures that the reduction is correct and parsimonious. Again, the same proof works if one replaces Symmetric by Skew-Symmetric and $\bar{C}$ by $C$.

6.4. Reduction from \#2D-X-RAY to \#(SkEW-)Symmetric 2D-X-RAY. The following Lemma 6.12 is the first known result that connects X-RAY problems with their symmetric counterparts. 


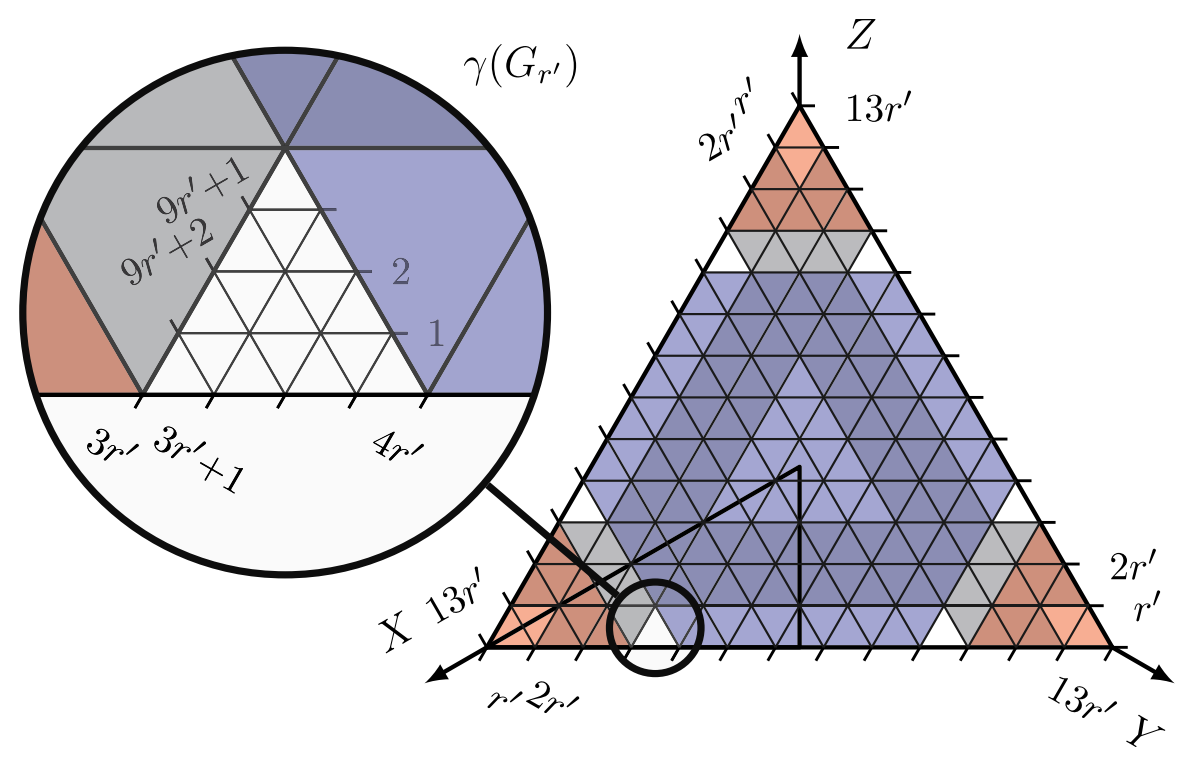

Figure 6.4: Visualizes the reduction from 2D-X-RAY to SYMMETRIC 2D-X-RAY. The lattice depicts the subdivision of the triangular grid $G_{r}$ into 13 regions per coordinate, each of width $r^{\prime}$. The intervals $\left(r^{\prime}, 3 r^{\prime}\right),\left(4 r^{\prime}, 9 r^{\prime}\right)$ and $\left(10 r^{\prime}, 13 r^{\prime}\right]$ - that is, the blocks of zeros in $\lambda$-are, respectively, indicated by gray-, blue- and redcolored areas; for instance, a point is colored gray if at least one of its coordinates lies in $\left(r^{\prime}, 3 r^{\prime}\right)$. Observe that the circled region equals the image of $G_{r^{\prime}}$ under $\gamma$, which is the only uncolored region intersecting the (closed) cone $\bar{C}$.

LEMMA 6.12. There exist parsimonious polynomial-time reductions from \#2D-X-RAY to \#SYMmetric 2D-X-RAY and to \#SKEW-SyMmetric 2D-X-RAY.

Proof. Let $\mu^{\prime}, \nu^{\prime}, \rho^{\prime} \in \mathbb{N}^{\left[0, r^{\prime}\right]}$ be a given \#2D-X-RAY instance. We start to construct the corresponding \#SYMMETRIC 2D-XRAY instance by choosing $r:=13 r^{\prime}$ and

$$
\lambda:=(\rho_{0}^{\prime}, \ldots, \rho_{r^{\prime}}^{\prime}, \underbrace{0, \ldots, 0}_{2 r^{\prime}-1}, \nu_{0}^{\prime}, \ldots, \nu_{r^{\prime}}^{\prime}, \underbrace{0, \ldots, 0}_{5 r^{\prime}-1}, \mu_{0}^{\prime}, \ldots, \mu_{r^{\prime}}^{\prime}, \underbrace{0, \ldots, 0}_{3 r^{\prime}}) ;
$$

notice that in fact $\lambda \in \mathbb{N}^{[0, r]}$. 
We claim that the \#SYmmetric 2D-X-RAY instance $\lambda$ has the same number of solutions as the given \#2D-X-RAY instance $\left(\mu^{\prime}, \nu^{\prime}, \rho^{\prime}\right)$. Indeed, consider the following map $\gamma$ that maps points in $G_{r^{\prime}}$ to points in $G_{r} \cap \bar{C}$ :

$$
\gamma(x, y, z)=\left(x+9 r^{\prime}, y+3 r^{\prime}, z\right)
$$

We lift $\gamma$ from points to point sets in the canonical way and abuse notation by calling the resulting map $\gamma$ again. We claim that $\gamma$ maps bijectively

$$
\gamma:\left\{\begin{array}{c}
\text { point sets in } G_{r^{\prime}} \text { with } \\
(X, Y, Z) \text {-marginal } \\
\left(\mu^{\prime}, \nu^{\prime}, \rho^{\prime}\right)
\end{array}\right\} \longrightarrow\left\{\begin{array}{c}
\text { point sets in } G_{r} \cap \bar{C} \\
\text { with sum-marginal } \lambda
\end{array}\right\},
$$

which then finishes the proof. An illustration of $\gamma$ is provided in Figure 6.4. The well-definedness and the injectivity of $\gamma$ are obvious. It remains to prove the surjectivity of $\gamma$. We prove surjectivity by constructing an explicit preimage. To achieve this, we start with some observations on the codomain of $\gamma$. So let $P$ be a point set in $G_{r} \cap \bar{C}$ with sum-marginal $\lambda$. Let $M:=\left[8 r^{\prime}, 9 r^{\prime}\right], N:=\left[3 r^{\prime}, 4 r^{\prime}\right]$ and $R:=\left[0, r^{\prime}\right]$. We first establish that for all points $(x, y, z) \in P$ it holds that $x, y$ and $z$ are all contained in $M, N$ or $R$ and neither two are included in the same interval. Since $\lambda_{i}=0$ for all $i \notin M \cup N \cup R$, it follows that $x, y$ and $z$ are indeed contained in $M \cup N \cup R$. In order to prove that the coordinates $x, y$ and $z$ must stem from pairwise distinct sets $M, N$ and $R$, we distinguish the following scenarios and show that each case causes a contradiction:

Two coordinates in $R$ : If, say, $x, y \in R$, then $z=r-x-y \geq$ $13 r^{\prime}-r^{\prime}-r^{\prime}=11 r^{\prime}$. But the range $\left[11 r^{\prime}, 13 r^{\prime}\right]$ intersects none of $M, N$ and $R$.

Two coordinates in $N$ : If, say, $x, y \in N$, then $z=r-x-y \geq$ $13 r^{\prime}-4 r^{\prime}-4 r^{\prime}=5 r^{\prime}$ and $z=r-x-y \leq 13 r^{\prime}-3 r^{\prime}-3 r^{\prime}=7 r^{\prime}$. But the range $\left[5 r^{\prime}, 7 r^{\prime}\right]$ intersects none of $M, N$ and $R$.

Two coordinates in $M$ : If, say, $x, y \in M$, then $z=r-x-y \leq$ $13 r^{\prime}-8 r^{\prime}-8 r^{\prime}<0$, which clearly contradicts $z \in\left[0,13 r^{\prime}\right]$.

We conclude that each element of $P$ is contained in $M \times N \times R$. Figure 6.4 visualizes this result geometrically: The remaining 6 
regions $\sigma(M \times N \times R)$ for permutations $\sigma \in \mathfrak{S}_{3}$ are precisely the 6 noncolored triangles, one of which lies in $\bar{C}$ (the left one at the very bottom). It now follows that the preimage of $P$ under $\gamma$ is the set $\left\{\left(x-9 r^{\prime}, y-3 r^{\prime}, z\right):(x, y, z) \in P\right\}$, which is a point set in $G_{r^{\prime}}$ with $(X, Y, Z)$-marginal $\left(\mu^{\prime}, \nu^{\prime}, \rho^{\prime}\right)$ as required.

Note that without any changes the whole proof also works for the open cone $C$ instead of $\bar{C}$, which proves the result for SkEwSymmetriC 2D-X-RAY.

The proofs of Theorem 3.5 and Theorem 3.6 are finished by combining the reductions in Lemmas 6.8, 6.9 and 6.12 with the hardness result in Gardner et al. (1999).

\section{Connection to Kronecker coefficients}

Problem 10 in Stanley (1999b). Let $V_{1}, V_{2}, V_{3}$ be finite dimensional complex vector spaces. Then the group $\mathrm{GL}\left(V_{1}\right) \times \mathrm{GL}\left(V_{2}\right) \times$ $\mathrm{GL}\left(V_{3}\right)$ acts linearly on the tensor product $V_{1} \otimes V_{2} \otimes V_{3}$ via

$$
\left(g_{1}, g_{2}, g_{3}\right)\left(v_{1} \otimes v_{2} \otimes v_{3}\right):=\left(g_{1} v_{1}\right) \otimes\left(g_{2} v_{2}\right) \otimes\left(g_{3} v_{3}\right)
$$

and linear continuation. Hence $\mathrm{GL}\left(V_{1}\right) \times \mathrm{GL}\left(V_{2}\right) \times \mathrm{GL}\left(V_{3}\right)$ also acts linearly on the symmetric power $\operatorname{Sym}^{N}\left(V_{1} \otimes V_{2} \otimes V_{3}\right)$, which decomposes into irreducibles

$$
\operatorname{Sym}^{n}\left(V_{1} \otimes V_{2} \otimes V_{3}\right)=\bigoplus_{\mu, \nu, \rho}\left(S^{\mu} V_{1} \otimes S^{\nu} V_{2} \otimes S^{\rho} V_{3}\right)^{\oplus k(\mu, \nu, \rho)} .
$$

These multiplicities $k(\mu, \nu, \rho)$ are called the Kronecker coefficients and have been the focus of numerous publications, out of which Briand et al. (2009); Bürgisser \& Ikenmeyer (2008); Ikenmeyer et al. (2017); Pak \& Panova (2017) study their complexity.

Problem 10 in Stanley (1999b), which is also known as the Kronecker problem, asks for a combinatorial description of $k(\mu, \nu, \rho)$ (see Section 1). Many publications give combinatorial interpretations of the Kronecker coefficients in special subcases Ballantine \& Orellana (2005); Blasiak (2012); Hayashi (2015); Ikenmeyer et al. (2017); Lascoux (1980); Liu (2017); Remmel (1992); Remmel \& Whitehead (1994); Rosas (2001), and hence, these publications make progress toward resolving the Kronecker problem. 
Connecting Problems 9 and 10 in Stanley (1999b). Problem 9 in Stanley (1999b) is asking for a combinatorial interpretation for $a_{\lambda}(n, m)$; this is also known as the plethysm problem. Much less is known about this question than about the Kronecker problem, although numerous algorithms exist to compute plethysm coefficients (see the references provided in Loehr \& Remmel 2011), and Kahle \& Michałek (2018) rules out certain approaches toward finding combinatorial formulas. We make progress on the plethysm problem by giving a combinatorial interpretation in an interesting subcase, see Theorem 7.1 below. We carefully mimic the proof technique in Ikenmeyer et al. (2017), where a combinatorial interpretation for the Kronecker coefficient is given in a subcase. Since we reach the same combinatorial interpretation, we conclude that the plethysm coefficient equals the Kronecker coefficient in this case. The detailed statement can be found in Theorem 7.1 below.

To see the connection, consider the net of reductions as depicted in Figure 6.2: First, observe that all reductions ultimately start from \#2D-X-RAY. Furthermore, any reduction between two tomography problems is parsimonious. In a similar sense, the reductions to computing the Kronecker or plethysm coefficients preserve the number of solutions, too, by guaranteeing that the respective coefficients precisely reflect the number of solutions to the corresponding tomography problem.

For this reason, we can start with an arbitrary 2D-X-RAY instance $\left(\mu^{\prime}, \nu^{\prime}, \rho^{\prime}\right)$ of, say, $N$ solutions. By applying the reductions step-by-step, we obtain coefficients $k(\mu, \nu, \rho)$ and $b_{\lambda}(n, 3)$ that both count the number of solutions to the original instance, hence $k(\mu, \nu, \rho)=N=b_{\lambda}(n, 3)$. By carefully inspecting the reductions, it is easy to verify the following theorem:

TheOREM 7.1. Let $\mu^{\prime}, \nu^{\prime}, \rho^{\prime} \in \mathbb{N}^{[0, r]}$ be compositions satisfying $\sum_{i=0}^{r} i \cdot\left(\mu_{i}^{\prime}+\nu_{i}^{\prime}+\rho_{i}^{\prime}\right)=r\left|\mu^{\prime}\right|=r\left|\nu^{\prime}\right|=r\left|\rho^{\prime}\right|$ (i.e., the corresponding $2 \mathrm{D}-\mathrm{X}-\mathrm{RAY}$ instance is not trivially unsatisfiable). Let

$$
\begin{aligned}
\mu & :=\left(\mu^{\prime}+X\left(Q_{r-1}\right)\right)^{t}, \\
\nu & :=\left(\nu^{\prime}+Y\left(Q_{r-1}\right)\right)^{t}, \\
\rho & :=\left(\rho^{\prime}+Z\left(Q_{r-1}\right)\right)^{t},
\end{aligned}
$$


where $Q_{r}:=\{(x, y, z): x+y+z \leq r\} \subseteq \mathbb{N}^{3}$. Moreover, let

$$
\begin{aligned}
& \lambda:=\left((\rho^{\prime}, \underbrace{0, \ldots, 0}_{2 r-1}, \nu^{\prime}, \underbrace{0, \ldots, 0}_{5 r-1}, \mu^{\prime}, \underbrace{0, \ldots, 0}_{3 r})+S\left(P_{13 r-1}\right)\right)^{t}, \\
& \pi:=(\rho^{\prime}, \underbrace{0, \ldots, 0}_{2 r-1}, \nu^{\prime}, \underbrace{0, \ldots, 0}_{5 r-1}, \mu^{\prime}, \underbrace{0, \ldots, 0}_{3 r})+S\left(\bar{P}_{13 r-1}\right) .
\end{aligned}
$$

Then $k(\mu, \nu, \rho)=a_{\lambda}(|\lambda| / 3,3)=b_{\pi}(|\pi| / 3,3)$.

To illustrate the application of Theorem 7.1, we give some brief examples starting with 2D-X-RAY instances of size $r=1$ each. Unfortunately, all such instances are either uniquely satisfiable or unsatisfiable and thus the resulting plethysm and Kronecker coefficients take values 0 and 1 only.

EXAMPLE 7.2. Consider the following 2D-X-RAY instance $\mu^{\prime}=$ $(1,1), \nu^{\prime}=(1,1)$ and $\rho^{\prime}=(2,0)$ for $r=1$, which is uniquely satisfiable by $\boldsymbol{\Lambda}=\{(1,0,0),(0,1,0)\}$.

Following Theorem 7.1, let $\mu=((1,1)+(3,1))^{t}=(4,2)^{t}=$ $(2,2,1,1), \nu=(2,2,1,1)$ and $\rho=(2,1,1,1,1)$. Moreover, since $S\left(P_{12}\right)=(30,26,22,19,16,13,11,9,6,4,2,1)$, we assign $\lambda=((2,0$, $\left.0,1,1,0,0,0,0,1,1)+S\left(P_{12}\right)\right)^{t}=(12,11,11,10,10,9,8,8,8,7,7,6$, $6,5,5,5,5,4,4,4,3,3,2,2,2,2,1,1,1,1,1,1)$. Then $k(\mu, \nu, \rho)=$ $a_{\lambda}(55,3)=1$.

EXAmPle 7.3. Consider the 2D-X-RAY instance $\mu^{\prime}=(2,1), \nu^{\prime}=$ $(2,1)$ and $\rho^{\prime}=(2,1)$ for $r=1$, which is again uniquely satisfiable by $\boldsymbol{\Lambda}=\{(1,0,0),(0,1,0),(0,1,0)\}$.

By choosing $\mu=(2,2,1,1,1), \nu=(2,2,1,1,1), \rho=(2,2,1,1,1)$ and $\pi=(2,1,0,2,1,0,0,0,0,2,1)+(61,54,46,38,31,23,17,12,9$, $6,4,2)=(63,55,46,40,32,23,17,12,9,8,5,2)$ as in Theorem 7.1, we find that $k(\mu, \nu, \rho)=b_{\pi}(104,3)=1$.

EXAmple 7.4. Consider the 2D-X-RAY instance $\mu^{\prime}=(2,0), \nu^{\prime}=$ $(2,0)$ and $\rho^{\prime}=(0,2)$ for $r=1$; it is easy to check that the instance is unsatisfiable.

We construct $\mu=(2,1,1,1,1), \nu=(2,1,1,1,1), \rho=(2,2,2)$ and $\pi=(0,2,0,2,0,0,0,0,0,2)+(61,54,46,38,31,23,17,12,9,6$, 
$4,2)=(61,56,46,40,31,23,17,12,9,8,4,2)$ as in Theorem 7.1. It follows that $k(\mu, \nu, \rho)=b_{\pi}(103,3)=0$.

\section{Positive formulas for restricted plethysm coefficients}

In the course of the main proof, we discovered that a restricted class of plethysm coefficients can be explained as the number of certain combinatorial objects. As motivated before, results of that sort are of independent interest.

Formally speaking, the general goal is to show that the problem of computing general plethysm coefficients $p_{\lambda}(\mu, \nu)$ is contained in the complexity class \#P. Completely resolving that hypothesis seems out of reach with today's techniques; nevertheless, we make partial progress by proving that for certain nontrivial sets of inputs $(\mu, \nu, \lambda)$, computing $p_{\lambda}(\mu, \nu)$ actually is in \#P. In particular, let

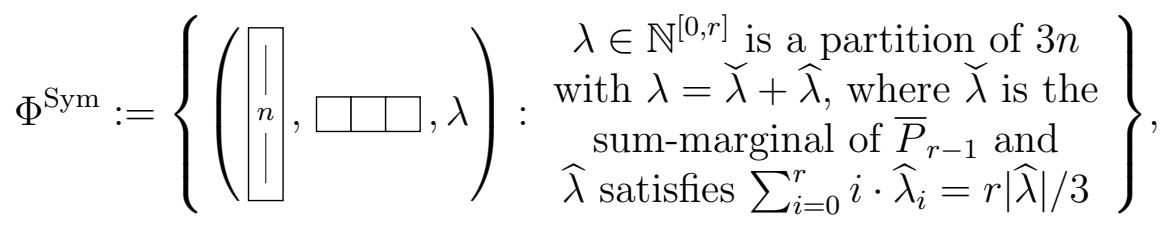

and

$$
\Phi^{\wedge}:=\left\{\left(\mid \begin{array}{l}
\| \\
\|
\end{array}, \square, \lambda\right) \begin{array}{c}
\lambda \in \mathbb{N}^{[0, r]} \text { is a partition of } 3 n \\
\text { with } \lambda=\widehat{\lambda}+\widehat{\lambda}, \text { where } \widetilde{\lambda} \text { is the } \\
\text { sum-marginal of } P_{r-1} \text { and } \\
\hat{\lambda}_{\text {satisfies } \sum_{i=0}^{r} i \cdot \widehat{\lambda}_{i}=r|\widehat{\lambda}| / 3}
\end{array}\right\} .
$$

The following theorem follows from Proposition 5.5.

THEOREM 8.1. The problem of computing plethysm coefficients $p_{\lambda}(\mu, \nu)$ is in \#P, when restricted to instances $(\mu, \nu, \lambda)$ in $\Phi^{\mathrm{Sym}}$ or $\Phi^{\wedge}$.

Using the same idea, we identify a more general class of instances $(\mu, \nu, \lambda)$ for which the same result applies. Namely, for any choice of $\mu$ (and $\nu=\square \square$ or $\nu=\square \square \square^{t}$ ), we can construct partitions $\lambda$ in the same spirit as above. Let $\bar{p}(r)=\left|\bar{P}_{r}\right|$ denote the 
number of points in the complete pyramid $\bar{P}_{r}$, or equivalently, the total number of partitions of all integers $\leq r$ into at most three parts. Any Young diagram $\mu=\left(n_{0}, n_{1}, \ldots, n_{\ell}\right)^{t}$ can be written as

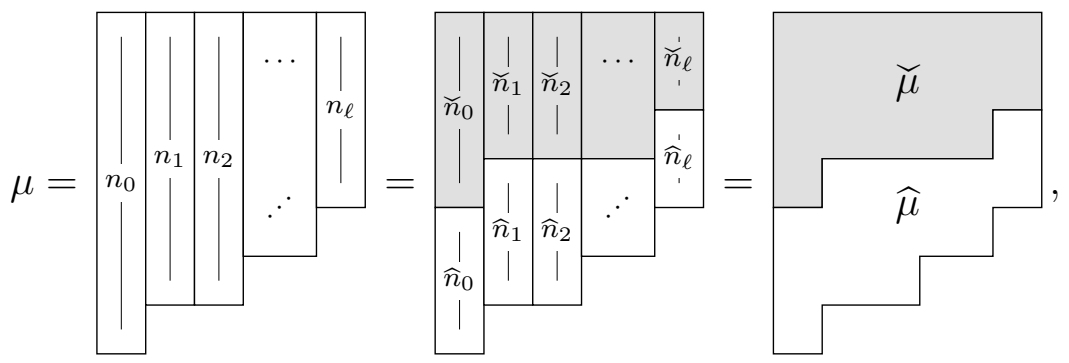

where, for all $0 \leq j \leq \ell$, we pick $r_{j}$ minimal so that $n_{j}<\bar{p}\left(r_{j}\right)$ and assign $\breve{n}_{j}:=\bar{p}\left(r_{j}-1\right)$ and $\widehat{n}_{j}:=n_{j}-\breve{n}_{j}$. Furthermore, let $n:=\sum_{j} n_{j}=\sum_{j} \breve{n}_{j}+\widehat{n}_{j}$. As depicted, we refer to the upper rows (that is, rows of small indices) by $\breve{\mu}$ and denote the remaining skew shape by $\widehat{\mu}$. After decomposing $\mu$ in that manner, let us define

$\Psi^{\text {Sym }}:=\left\{(\mu, \square \square, \lambda): \begin{array}{c}\lambda \in \mathbb{N}^{\left[0, r_{0}\right]} \text { is a partition of } 3 n \\ \text { with } \lambda=\sum_{j} \widetilde{\lambda}^{j}+\widehat{\lambda}^{j}, \text { where } \widehat{\lambda}^{j} \text { is } \\ \text { the sum-marginal of } \bar{P}_{r_{j}-1} \text { and } \\ \widehat{\lambda}^{j} \text { satisfies } \sum_{i=0}^{r_{j}} i \cdot \widehat{\lambda}_{i}^{j}=\widehat{n}_{j} \cdot r_{j}\end{array}\right\}$.

In a very similar way, we can define $\Psi^{\wedge}$ : This time, let $p(r)$ be the total number of partitions of all integers $\leq r$ into at most three distinct parts (that is, the number of points in the complete pyramid $P_{r}$ ). Given $\mu$, determine $r_{0}, \ldots, r_{\ell}$ as before with $p(\cdot)$ in place of $\bar{p}(\cdot)$. Then:

$$
\Psi^{\wedge}:=\left\{(\mu, \boxminus, \lambda): \begin{array}{c}
\lambda \in \mathbb{N}^{\left[0, r_{0}\right]} \text { is a partition of } 3 n \\
\text { with } \lambda=\sum_{j} \widetilde{\lambda}^{j}+\widehat{\lambda}^{j}, \text { where } \widetilde{\lambda}^{j} \text { is } \\
\text { the sum-marginal of } P_{r_{j}-1} \text { and } \\
\widehat{\lambda}^{j} \text { satisfies } \sum_{i=0}^{r_{j}} i \cdot \widehat{\lambda}_{i}^{j}=\widehat{n}_{j} \cdot r_{j}
\end{array}\right\} .
$$

THEOREM 8.2. The problem of computing plethysm coefficients $p_{\lambda}(\mu, \nu)$ is in \#P, when restricted to instances $(\mu, \nu, \lambda)$ in $\Psi^{\text {Sym }}$ or $\Psi^{\wedge}$. 
Clearly, $\Phi^{\text {Sym }} \subset \Psi^{\text {Sym }}$ and $\Phi^{\wedge} \subset \Psi^{\wedge}$, so Theorem 8.2 really generalizes Theorem 8.1. For the following proof of Theorem 8.2, we will omit the treatment of $\Psi \wedge$ and focus on $\Psi^{\text {Sym }}$ only; both arguments are analogous and the only exceptions have been pointed out in the preceding sections.

We catch up on some representation theoretic background. A Young tableau $T$ is a Young diagram $\lambda$ together with a filling of all boxes in $\lambda$ with objects taken from some alphabet. If a total ordering on the alphabet is understood, then we say that $T$ is semistandard whenever the entries of $T$ are strictly increasing down each column and weakly increasing along each row. The Schur module $S^{\lambda} V$ can explicitly be constructed in terms of Young tableaux: By arbitrarily indexing the boxes of $\lambda$, we fix a basis $\left\{v_{T}\right\}_{T}$ for $\boldsymbol{\bigotimes}^{|\lambda|} V$ where $T$ ranges over all Young tableaux of shape $\lambda$ filled with basis elements of $V$. Now $S^{\lambda} V$ is the largest subspace of $\otimes^{|\lambda|} V$ satisfying the following two exchange conditions (known as the Grassmann-Plücker relations):

1. $v_{T}=-v_{T^{\prime}}$ where $T^{\prime}$ is obtained from $T$ by exchanging two vertically adjacent boxes.

2. $v_{T}=\sum_{T^{\prime}} v_{T^{\prime}}$ where, for some $i$ and $\ell, T^{\prime}$ ranges over all tableaux obtained from $T$ by exchanging the top-most $\ell$ boxes in column $i$ with any $\ell$ boxes in column $i-1$ preserving the vertical order.

It is known that the set of all semistandard tableaux of shape $\lambda$ filled with basis elements of $V$ forms a basis of $S^{\lambda} V$.

For the remainder of this section, let $(\mu, \nu, \lambda) \in \Psi^{\mathrm{Sym}}$ and let $X_{0}, X_{1}, \ldots, X_{k-1}$ be an ordered basis of $V=\mathbb{C}^{k}$. Then the set of all monomials $X_{x} X_{y} X_{z}$ for $x, y, z \in[0, k-1]$ forms a basis of $\operatorname{Sym}^{3} V$; we sometimes identify monomials $X_{x} X_{y} X_{z}$ with their representatives $(x, y, z) \in \bar{C}_{<k}:=[0, k-1]^{3} \cap \bar{C}$. Next, let $\prec$ be the partial ordering on $\bar{C}_{<k}$ defined by $(x, y, z) \prec\left(x^{\prime}, y^{\prime}, z^{\prime}\right)$ whenever $x+y+z<x^{\prime}+y^{\prime}+z^{\prime}$ and arbitrarily extend $\prec$ to a total order. From the previous paragraph, we derive that $\left\{v_{T}\right\}_{T}$ forms a basis of $S^{\mu} S^{\nu} V=S^{\mu} \operatorname{Sym}^{3} V$, where $T$ ranges over all semistandard tableaux of shape $\mu$ and alphabet $\bar{C}_{<k}$. By construction, the weight of a vector $v_{T}$ equals the sum of all entry-wise weights in $T$. 
For a Young tableau $T$ of shape $\mu$, we write $\breve{T}$ to denote the subtableau of shape $\breve{\mu}$ and we write $\widehat{T}$ to denote the skew tableau corresponding to $\widehat{\mu}$.

We proceed to rework some parts of Sections 5 and6, where we now consider semistandard tableaux in place of pyramids - the exact same proof is recovered when restricting $\mu$ (and thereby the shape of the tableaux $T$ ) to a single column.

Lemma 8.3. Let $T$ be a semistandard Young tableau of shape $\mu$ and weight $\lambda$. Then:

1. $\breve{T}$ is of weight $\breve{\lambda}:=\sum_{j} \breve{\lambda}^{j}$. In fact, there exists only one such tableau $\breve{T}$, which is obtained by filling all boxes in the $i$-th row of $\breve{\mu}$ with the $i$-th smallest monomial according to $\prec$.

2. $\widehat{T}$ is of weight $\widehat{\lambda}:=\sum_{j} \widehat{\lambda}^{j}$. Moreover, the $j$-th column of $\widehat{T}$ exclusively contains monomials $X_{x} X_{y} X_{z}$ where $x+y+z=r_{j}$.

The proof of Lemma 8.3 roughly follows Lemma 6.11, and to this end we first lift the definition of coordinate sums to tableaux: Let

$$
B(T)=\sum_{(x, y, z)} x+y+z,
$$

where the sum is over all entries $(x, y, z)$ of $T$. Again, it is easy to relate the coordinate sum of $T$ with its weight: $B(T)=\sum_{i=0}^{r} i \cdot \kappa_{i}$, where $\kappa \in \mathbb{N}^{[0, r]}$ is the weight of $T$.

Proof of Lemma 8.3. Let $T$ be a semistandard Young tableau of shape $\mu$ and weight $\lambda$. We determine $B(T)$ exactly:

$$
\begin{aligned}
B(T)=\sum_{i=0}^{r_{0}} i \cdot \lambda_{i} & =\sum_{j=0}^{\ell}\left(\sum_{i=0}^{r_{j}} i \cdot \check{\lambda}_{i}^{j}+\sum_{i=0}^{r_{j}} i \cdot \widehat{\lambda}_{i}^{j}\right) \\
& =\sum_{j=0}^{\ell}\left(\sum_{i=0}^{r_{j}} i \cdot \breve{\lambda}_{i}^{j}+\widehat{n}_{j} \cdot r_{j}\right) .
\end{aligned}
$$

Recall that $\breve{\lambda}^{j}$ equals the sum-marginal of the complete pyramid $\bar{P}_{r_{j}}$. In viewing the $j$-th column in $T$ as a point set in the closed 
cone of size $n_{j}=\left|\bar{P}_{r_{j}}\right|+\widehat{n}_{j}$, Lemma 6.10 yields that the contribution of the $j$-th column to the coordinate sum $B(T)$ is at least $\sum_{i=0}^{r_{j}} i \cdot \breve{\lambda}_{i}^{j}+\widehat{n}_{j} \cdot r_{j}$. In conjunction with (8.4), that bound is tight. By applying Lemma 6.10 again, we learn that for all $j$, the $j$-th column of $T$ corresponds to a pyramid $P$ that satisfies $\bar{P}_{r_{j}-1} \subseteq P \subset \bar{P}_{r_{j}}$. But then the only way to label the boxes in the $j$-th column of $T$ is as stated: The top-most $\bar{n}_{j}$ boxes are filled with points in $\bar{P}_{r_{j}-1}$ and all $\widehat{n}_{j}$ boxes below are filled with points in $G_{r_{j}} \cap \bar{C}$.

LEMma 8.5. Let $T$ be a semistandard Young tableau of shape $\mu$ and weight $\lambda$. Then $v_{T}$ is a highest weight vector in $S^{\mu} \mathrm{Sym}^{3} V$.

Proof. We assert that $v_{T}$ vanishes under all raising operators $E_{a, b}$. In fact, it suffices to show that $v_{T}$ vanishes under $E_{a, b}$ after canonically embedding $v_{T} \in S^{\mu} \operatorname{Sym}^{3} V \subset \bigotimes_{j=0}^{\ell} \bigwedge^{n_{j}} \operatorname{Sym}^{3} V$ (that is, the space obtained by omitting condition 2 in the above characterization of Schur modules). Let

$$
\varphi: \bigotimes_{j=0}^{\ell} \bigwedge^{n_{j}} \operatorname{Sym}^{3} V \rightarrow S^{\mu} \operatorname{Sym}^{3} V
$$

be the canonical projection. We rewrite $v_{T}=\varphi\left(v_{T_{0}} \otimes \ldots \otimes v_{T_{\ell}}\right)$, where each column $T_{j}$ of $T$ is interpreted as a point set in the closed cone and $v_{P}$ for $P$ such a point set is chosen as in Theorem 5.3. Lemma 8.3 in particular implies that each column $T_{j}$ is a pyramid in the closed cone, which entails that $E_{a, b} v_{T_{j}}=0$ by Theorem 5.3. Hence:

$$
\begin{aligned}
E_{a, b} v_{T} & =E_{a, b} \varphi\left(v_{T_{0}} \otimes \cdots \otimes v_{T_{\ell}}\right) \\
& =\varphi\left(E_{a, b} v_{T_{0}} \otimes \cdots \otimes v_{T_{\ell}}\right)+\cdots+\varphi\left(v_{T_{0}} \otimes \cdots \otimes E_{a, b} v_{T_{\ell}}\right)=0 ;
\end{aligned}
$$

the second equality holds since $\varphi$ is $\mathrm{GL}(V)$-equivariant.

Recall that $p_{\lambda}(\mu, \nu)$ equals the dimension of the weight- $\lambda$ highest weight subspace of $S^{\mu} S^{\nu} V$. Since each elementary weight- $\lambda$ vector is a highest weight vector in this case, $p_{\lambda}(\mu, \nu)$ equals the dimension of the weight subspace of weight $\lambda$ - or equivalently, 
the number of semistandard tableaux of shape $\mu$ and weight $\lambda$. Formally, we obtain the following statement which implies Theorem 8.2 as an immediate consequence.

Lemma 8.6. Let $(\mu, \nu, \lambda) \in \Psi^{\mathrm{Sym}}$. Then $p_{\lambda}(\mu, \nu)$ equals the number of semistandard tableaux of shape $\mu$ and weight $\lambda$, where all boxes are filled with points in $\bar{C}_{<k}$.

\section{GapP-Completeness}

This section is devoted to proving Theorem 3.8, which claims that computing general plethysm coefficients $p_{\lambda}(\mu, \nu)$, and also the special cases $a_{\lambda}(n, m)$ and $b_{\lambda}(n, m)$, is GapP-complete. The hardness part follows immediately from Theorem $3.6^{7}$. In order to show containment in GapP, we will derive an explicit formula involving signs for the general plethysm coefficients $p_{\lambda}(\mu, \nu)$. We heavily rely on tools from the theory of symmetric functions; however, we shall refrain from describing the necessary background in detail and instead limit ourselves to the most important definitions; see for instance Stanley (1999a) for a thorough introduction. The following argument is mostly standard and similar to, e.g., Drfler et al. (2020).

Let $\Lambda$ denote the ring of symmetric polynomials (that is, polynomials which are invariant under any permutation of variables) in finitely many variables $X_{0}, X_{1}, \ldots$. The characters of the irreducible $\mathrm{GL}_{n}$-representation $S^{\nu} \mathbb{C}^{n}$ are elements $s_{\nu}\left(X_{0}, \ldots, X_{n-1}\right) \in$ $\Lambda$ called Schur functions (where $X_{0}, \ldots, X_{n-1}$ correspond to the eigenvalues of the conjugacy class of $\mathrm{GL}_{n}$ ). Recall the notion of semistandard Young tableaux as introduced in Section 8 and let $T_{0}, \ldots, T_{l-1}$ be an arbitrary but exhaustive enumeration of all semistandard tableaux of shape $\nu$ filled with entries $0, \ldots, n-1$. The

\footnotetext{
${ }^{7}$ Indeed, it is folklore that under the appropriate notions of reductions which allow for pre- and post-computations, \#P-hardness implies GapP-hardness. To see this, let $f$ be \#P-hard and let $g_{1}-g_{2} \in \mathrm{GapP}$ so that $g_{1}, g_{2} \in \# \mathrm{P}$. The trick is to construct a nonnegative function $g \in \# \mathrm{P}$ by encoding the nonnegative functions $g_{1}, g_{2}$ in different blocks of bits. Now, since $f$ is \#P-hard, there exists a polynomial-time reduction from $g$ to $f$ and we can use the post-processing step to recover the intended function value $g_{1}-g_{2}$.
} 
weight $\operatorname{wt}(T) \in \mathbb{N}^{[0, n-1]}$ of a tableau $T$ is defined as the composition such that $\operatorname{wt}(T)_{i}$ is the number of entries $i$ in $T$. We have that

$$
s_{\nu}\left(X_{0}, \ldots, X_{n-1}\right)=\sum_{i=0}^{l-1} X^{T_{i}},
$$

for $X^{T}:=\prod_{t \in T} X_{t}$, where the product is over (the multiset of) the entries $t$ of $T$. Following this description, it follows that the character of the composed representation $S^{\mu} S^{\nu} \mathbb{C}^{n}$ is given by

$$
s_{\mu}\left[s_{\nu}\right]\left(X_{0}, \ldots, X_{n-1}\right):=s_{\mu}\left(X^{T_{0}}, \ldots, X^{T_{l-1}}\right) ;
$$

that composition $s_{\mu}\left[s_{\nu}\right]$ of symmetric functions is called a symmetric function plethysm.

The set of Schur function forms a basis of $\Lambda$. Moreover, since the Schur functions are the characters of the irreducible $\mathrm{GL}_{n}$-representations, there exists an inner product $\langle\cdot, \cdot \cdot\rangle$ on $\Lambda$ so that the Schur functions form an orthonormal basis: $\left\langle s_{\lambda}, s_{\mu}\right\rangle=\delta_{\lambda \mu}$. It follows that for any $\mathrm{GL}_{n}$-representation with character $\chi$, the multiplicity of $S^{\lambda} \mathbb{C}^{n}$ in its decomposition into irreducibles equals $\left\langle s_{\lambda}, \chi\right\rangle$. In particular, $p_{\lambda}(\mu, \nu)=\left\langle s_{\lambda}, s_{\mu}\left[s_{\nu}\right]\right\rangle$. It remains to develop a GapPformula for $\left\langle s_{\lambda}, s_{\mu}\left[s_{\nu}\right]\right\rangle$.

As a special case of Schur functions, the complete homogeneous symmetric functions $h_{d}$ are defined as $s_{(d)}$; we write $h_{\lambda}:=$ $h_{\lambda_{0}} h_{\lambda_{1}} \ldots$. Moreover, let $m_{\lambda}:=\sum_{\rho \in \mathfrak{S}_{n}(\lambda)} X^{\rho}$ denote the monomial symmetric functions, where $\mathfrak{S}_{n}(\lambda)$ is the set of all (distinct) permutations of $\lambda=\left(\lambda_{0}, \ldots, \lambda_{n-1}\right)$ and $X^{\rho}:=\prod_{i} X_{i}^{\rho_{i}}$. The wellknown Jacobi-Trudi identity (Stanley 1999a, Sec. 7.16) expresses any Schur function as a determinant:

$$
s_{\lambda}=\operatorname{det}\left(h_{\lambda_{i}-i+j}\right)_{i, j=0}^{\ell(\lambda)-1},
$$

thus $s_{\lambda}$ can be written as a signed sum of polynomials $h_{\mu}$. In addition, it is known that the orthogonal dual basis for the complete homogeneous symmetric functions is given by the monomial symmetric functions, i.e., $\left\langle h_{\lambda}, m_{\mu}\right\rangle=\delta_{\lambda \mu}$. Now suppose that $s_{\mu}\left[s_{\nu}\right]$ can be expressed as

$$
s_{\mu}\left[s_{\nu}\right]=\sum_{\kappa} q_{\kappa}(\mu, \nu) m_{\kappa},
$$


for some GapP-computable coefficients $q_{\kappa}(\mu, \nu)$. By the JacobiTrudi identity (9.2), we have

$$
\begin{aligned}
p_{\lambda}(\mu, \nu) & =\left\langle\operatorname{det}\left(h_{\lambda_{i}-i+j}\right)_{i, j=0}^{\ell(\lambda)-1}, s_{\mu}\left[s_{\nu}\right]\right\rangle \\
& =\sum_{\sigma \in \mathfrak{S}_{\ell(\lambda)}} \operatorname{sign}(\sigma) q_{\lambda-(0, \ldots, \ell(\lambda)-1)+\sigma}(\mu, \nu),
\end{aligned}
$$

where the permutation $\sigma$ is viewed as a vector with entries $0, \ldots$, $\ell(\lambda)-1$. This formula implies in particular that also $p_{\lambda}(\mu, \nu)$ can be computed by a GapP machine.

It now suffices to prove that the coefficients $q_{\kappa}(\mu, \nu)$ are indeed expressible as a GapP formula. From (9.1), one can derive that

$$
s_{\mu}=\sum_{\pi} K_{\mu \pi} m_{\pi}
$$

where $K_{\mu \pi}$ denotes the number of semistandard tableaux of shape $\mu$ and weight $\pi$ (called the Kostka coefficient). We finally obtain:

$$
\begin{aligned}
s_{\mu}\left[s_{\nu}\right]\left(X_{0}, \ldots, X_{n-1}\right) & =s_{\mu}\left(X^{T_{0}}, \ldots, X^{T_{l-1}}\right) \\
& =\sum_{\pi} K_{\mu \pi} m_{\pi}\left(X^{T_{0}}, \ldots, X^{T_{l-1}}\right) \\
& =\sum_{\pi} K_{\mu \pi} \sum_{\rho \in \mathfrak{G}_{l}(\pi)} X^{\sum_{i} \rho_{i} \operatorname{wt}\left(T_{i}\right)},
\end{aligned}
$$

and therefore

$q_{\kappa}(\mu, \nu)=\left\langle h_{\kappa}, s_{\mu}\left[s_{\nu}\right]\right\rangle=\sum_{\pi} K_{\mu \pi}\left|\left\{\rho \in \mathfrak{S}_{l}(\pi): \kappa=\sum_{i} \rho_{i} \operatorname{wt}\left(T_{i}\right)\right\}\right|$.

From that identity, it is obvious that $q_{\kappa}(\mu, \nu)$ is GapP-computable (even \#P-computable): The coefficients $K_{\mu \pi}$ are clearly \#P-computable and after guessing $\pi$ and $\rho$, we can check the condition $\kappa=\sum_{i} \rho_{i} \operatorname{wt}\left(T_{i}\right)$ in polynomial time.

\section{Acknowledgements}

This research was partially carried out when CI was at the Max Planck Institute for Software Systems, Saarbrücken. The author 
acknowledges support from the DFG grant IK 116/2-1.

Funding Open Access funding enabled and organized by Projekt DEAL.

Open Access This article is licensed under a Creative Commons Attribution 4.0 International License, which permits use, sharing, adaptation, distribution and reproduction in any medium or format, as long as you give appropriate credit to the original author(s) and the source, provide a link to the Creative Commons licence, and indicate if changes were made. The images or other third party material in this article are included in the article's Creative Commons licence, unless indicated otherwise in a credit line to the material. If material is not included in the article's Creative Commons licence and your intended use is not permitted by statutory regulation or exceeds the permitted use, you will need to obtain permission directly from the copyright holder. To view a copy of this licence, visit http://creativecommons.org/ licenses/by/4.0/.

\section{Publisher's Note}

Springer Nature remains neutral with regard to jurisdictional claims in published maps and institutional affiliations.

\section{References}

Cristina M. Ballantine \& Rosa C. Orellana (2005). A combinatorial interpretation for the coefficients in the Kronecker product $s_{(n-p, p)} * s_{\lambda}$. Sém. Lothar. Combin. 54A, Art. B54Af, 29 pp. (electronic).

Markus Bläser \& Christian Ikenmeyer (2018). Introduction to geometric complexity theory. Lecture notes, http://pcwww.liv. ac.uk/ iken/teaching_sb/summer17/introtogct/gct.pdf, version from July 25 .

Jonah Blasiak (2012). Kronecker coefficients for one hook shape. arXiv:1209.2018.

Emmanuel Briand, Rosa Orellana \& Mercedes H. Rosas (2009). Reduced Kronecker Coefficients and Counter-Examples to Mul- 
muley's Strong Saturation Conjecture SH. Computational Complexity $\mathbf{1 8}(4), 577$.

Sara Brunetti, Alberto Del Lungo \& Yan Grard (2001). On the computational complexity of reconstructing three-dimensional lattice sets from their two-dimensional X-rays. Linear Algebra and its Applications 339(1), 59-73.

Peter Bürgisser (2016). Permanent versus determinant, obstructions, and Kronecker coefficients. Séminaire Lotharingien de Combinatoire 75, 1-19. Article B75a.

Peter Bürgisser, Jesko Hüttenhain \& Christian Ikenmeyer (2017). Permanent versus determinant: Not via saturations. Proceedings of the American Mathematical Society 145, 1247-1258.

Peter Bürgisser \& Christian Ikenmeyer (2008). The complexity of computing Kronecker coefficients. In FPSAC 2008, Valparaiso-Viña del Mar, Chile, DMTCS proc. AJ, 357-368.

Peter Bürgisser \& Christian Ikenmeyer (2011). Geometric Complexity Theory and Tensor Rank. In Proceedings of the 43rd Annual ACM Symposium on Theory of Computing, STOC 2011, 509-518. ACM, New York, NY, USA.

Peter Bürgisser \& Christian Ikenmeyer (2013). Deciding positivity of Littlewood-Richardson coefficients. SIAM Journal of Discrete Mathematics 27, 1639-1681.

Peter Bürgisser \& Christian Inenmeyer (2013). Explicit Lower Bounds via Geometric Complexity Theory. In Proceedings of the 45th Annual ACM Symposium on Theory of Computing, STOC 2013, 141150. ACM, New York, NY, USA.

Peter Bürgisser, Christian Ikenmeyer \& Greta Panova (2019). No occurrence obstructions in geometric complexity theory. Journal of the American Mathematical Society 32, 163-193.

Peter Bürgisser, Joseph M. Landsberg, Laurent Manivel \& JERZY WEYMAN (2011). An overview of mathematical issues arising in the geometric complexity theory approach to VP v.s. VNP. SIAM J. Comput. 40(4), 1179-1209. 
Christophe CARr (1990). Plethysm of elementary functions. Bayreuther Mathematische Schriften 31, 1-18.

Matthias Christandl, Brent Doran, Stavros Kousidis \& Michael Walter (2014). Eigenvalue Distributions of Reduced Density Matrices. Communications in Mathematical Physics 332(1), 1-52.

Matthias Christandl, Aram W. Harrow \& Graeme Mitchison (2007). Nonzero Kronecker Coefficients and What They Tell us about Spectra. Communications in Mathematical Physics 270, 575-585.

Jesús A. De Loera \& Tyrrell B. McAllister (2006). On the computation of Clebsch-Gordan coefficients and the dilation effect 15(1), $7-19$.

Julian Drfler, Christian Ikenmeyer \& Greta Panova (2020). On Geometric Complexity Theory: Multiplicity Obstructions Are Stronger Than Occurrence Obstructions. SIAM Journal on Applied Algebra and Geometry 4(2), 354-376. Conference version in the Proceedings of the 46th International Colloquium on Automata, Languages and Programming, 2019.

Stephen A. Fenner, Lance J. Fortnow \& Stuart A. Kurtz (1994). Gap-definable counting classes. Journal of Computer and System Sciences 48(1), 116-148.

H. O. Foulkes (1950). Concomitants of the Quintic and Sextic Up To Degree Four in the Coefficients of the Ground Form. Journal of the London Mathematical Society s1-25(3), 205-209.

William Fulton \& Joe Harris (1991). Representation Theory - A First Course, volume 129 of Graduate Texts in Mathematics. Springer.

Richard J. Gardner, Peter Gritzmann \& Dieter PrangenBERG (1999). On the Computational Complexity of Reconstructing Lattice Sets from Their X-rays. Discrete Math. 202(1-3), 45-71.

Fulvio Gesmundo, Christian Ikenmeyer \& Greta Panova (2017). Geometric complexity theory and matrix powering. Differential Geometry and its Applications 55, 106-127. Part of special issue: Geometry and complexity theory. 
TAKahiro Hayashi (2015). A decomposition rule for certain tensor product representations of the symmetric groups. arXiv:1507.02047.

Charles Hermite (1854). Sur la theorie des fonctions homogenes à deux indéterminées. Cambridge and Dublin Mathematical Journal 9, 172217 .

Christian Inenmeyer (2012). Geometric Complexity Theory, Tensor Rank, and Littlewood-Richardson Coefficients. Ph.D. thesis, Institute of Mathematics, University of Paderborn. Online available at http:// nbn-resolving.de/urn:nbn:de:hbz:466:2-10472.

Christian Ikenmeyer (2019). GCT and symmetries. http://pcwww . liv.ac.uk/ iken/teaching_sb/winter1718/gct2/symmetries.pdf, version from January 30, 2019.

Christian Ikenmeyer \& Umangathan Kandasamy (2020). Implementing Geometric Complexity Theory: On the Separation of Orbit Closures via Symmetries. In Proceedings of the 52nd Annual ACM SIGACT Symposium on Theory of Computing, STOC 2020, 713726. Association for Computing Machinery.

Christian Ikenmeyer, Ketan D. Mulmuley \& Michael Walter (2017). On Vanishing of Kronecker Coefficients. Comput. Complex. 26(4), 949-992.

Christian Inenmeyer \& Greta Panova (2017). Rectangular Kronecker coefficients and plethysms in geometric complexity theory. $A d$ vances in Mathematics 319, 40-66.

Harlan Kadish \& Joseph M. Landsberg (2014). Padded Polynomials, Their Cousins, and Geometric Complexity Theory. Communications in Algebra 42(5), 2171-2180.

Thomas Kahle \& Mateusz Michąek (2016a). Plethysm and Lattice Point Counting. Foundations of Computational Mathematics 16, 12411261.

Thomas Kahle \& Mateusz Michąek (2016b). Plethysm and Lattice Point Counting. arXiv:1408.5708v3. This preprint version extends the version in Foundations of Computational Mathematics. 
Thomas Kahle \& Mateusz MichąeK (2018). Obstructions to Combinatorial Formulas for Plethysm. The Electronic Journal of Combinatorics $\mathbf{2 5}$.

Kazufumi Kimoto \& Soo Teck Lee (2019). Highest Weight Vectors in Plethysms. Communications in Mathematical Physics .

Hanspeter Kraft (1985). Geometrische Methoden in der Invariantentheorie. Friedr. Vieweg und Sohn Verlagsgesellschaft, Braunschweig. ISBN 3-528-18525-2.

ShraWAn Kumar (2015). A study of the representations supported by the orbit closure of the determinant. Compositio Mathematica 151(2), 292-312.

Joseph Landsberg (2011). Tensors: Geometry and Applications, volume 128 of Graduate Studies in Mathematics. American Mathematical Society, Providence, Rhode Island. ISBN 978-0-8218-6907-9.

Joseph M. Landsberg (2017). Geometry and Complexity Theory. Cambridge Studies in Advanced Mathematics. Cambridge University Press.

Alain Lascoux (1980). Produit de Kronecker des représentations du groupe symétrique. In Séminaire d'Algèbre Paul Dubreil et Marie-Paule Malliavin, 32ème année (Paris, 1979), volume 795 of Lecture Notes in Math., 319-329. Springer, Berlin.

RickY I. LiU (2017). A simplified Kronecker rule for one hook shape. Proc. Amer. Math. Soc. 145, 3657-3664.

Nicholas Loehr \& Jeffrey Remmel (2011). A computational and combinatorial exposé of plethystic calculus. Journal of Algebraic Combinatorics 33, 163-198.

Laurent Manivel (1998). Gaussian Maps and Plethysm. Algebraic Geometry (Catania, 1993 and Barcelone, 1994), Lecture Notes in Pure and Appl. Math. 200, 91-117.

Laurent Manivel (2011). On rectangular Kronecker coefficients. Journal of Algebraic Combinatorics 33(1), 153-162. 
Laurent Manivel \& Mateusz Michąek (2015). Secants of minuscule and cominuscule minimal orbits. Linear Algebra and its Applications 481, 288-312.

Ketan D. Mulmuley (2007). Geometric Complexity Theory VII: Nonstandard quantum group for the plethysm problem. arXiv:0709.0749.

Ketan D. Mulmuley (2009). Geometric Complexity Theory VI: The flip via saturated and positive integer programming in representation theory and algebraic geometry. arXiv:0704.0229v4.

Ketan D. Mulmuley, Hariharan Narayanan \& Milind Sohoni (2012). Geometric complexity theory III: On deciding nonvanishing of a Littlewood-Richardson coefficient. Journal of Algebraic Combinatorics 36(1), 103-110.

Ketan D. Mulmuley \& Milind Sohoni (2001). Geometric complexity theory I: An approach to the P vs. NP and related problems. SIAM J. Comput. 31(2), 496-526.

Ketan D. Mulmuley \& Milind Sohoni (2008). Geometric complexity theory II: Towards explicit obstructions for embeddings among class varieties. SIAM J. Comput. 38(3), 1175-1206.

Hariharan Narayanan (2006). On the complexity of computing Kostka numbers and Littlewood-Richardson coefficients. Journal of Algebraic Combinatorics 24(3), 347-354.

Igor PaK \& Greta Panova (2017). On the complexity of computing Kronecker coefficients. Comput. Complex. 26(1), 1-36.

Jeffrey B. Remmel (1992). Formulas for the expansion of the Kronecker products $S_{(m, n)} \otimes S_{\left(1^{p-r}, r\right)}$ and $S_{\left(1^{k} 2^{l}\right)} \otimes S_{\left(1^{p-r}, r\right)}$. Discrete Math. 99(1-3), 265-287.

Jeffrey B. Remmel \& Tamsen Whitehead (1994). On the Kronecker product of Schur functions of two row shapes. Bull. Belg. Math. Soc. Simon Stevin 1(5), 649-683.

Mercedes H. Rosas (2001). The Kronecker product of Schur functions indexed by two-row shapes or hook shapes. J. Algebraic Combin. 14(2), 153-173. 
Bruce E. SAGAN (2001). The symmetric group: Representations, combinatorial algorithms, and symmetric functions, volume 203 of Graduate Texts in Mathematics. Springer, New York, 2nd edition. ISBN 0-38795067-2.

Richard P. Stanley (1999a). Enumerative Combinatorics: Volume 2. Cambridge Studies in Advanced Mathematics. Cambridge University Press. ISBN 9780521560696.

Richard P. Stanley (1999b). Positivity Problems and Conjectures in Algebraic Combinatorics. In Mathematics: Frontiers and Perspectives, 295-319. American Mathematical Society.

Jean-Yves Thibon \& Christophe Carré (1992). Plethysm and vertex operators. Advances in applied Mathematics 13, 390-403.

Steven H. Weintraub (1990). Some observations on plethysms. Journal of Algebra 129, 103-114.

Jerzy Weyman (2003). Cohomology of Vector Bundles and Syzygies. Cambridge Tracts in Mathematics. Cambridge University Press.

Manuscript received 9 May 2020

NiCK FisCheR

Max Planck Institute for Informatics, Saarbrücken, Germany nfischer@mpi-inf .mpg.de

\section{Christian IKenmeyer}

The University of Liverpool, Liverpool, UK christian.ikenmeyer@ liverpool.ac.uk 